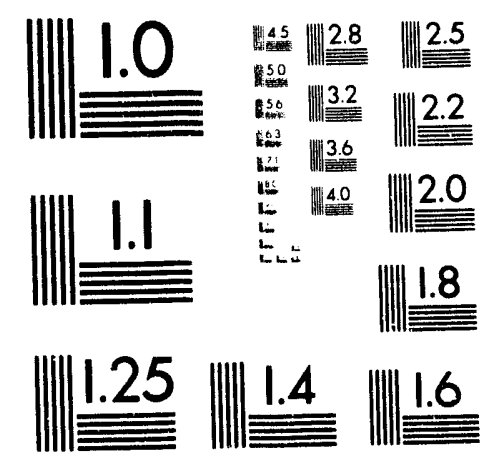



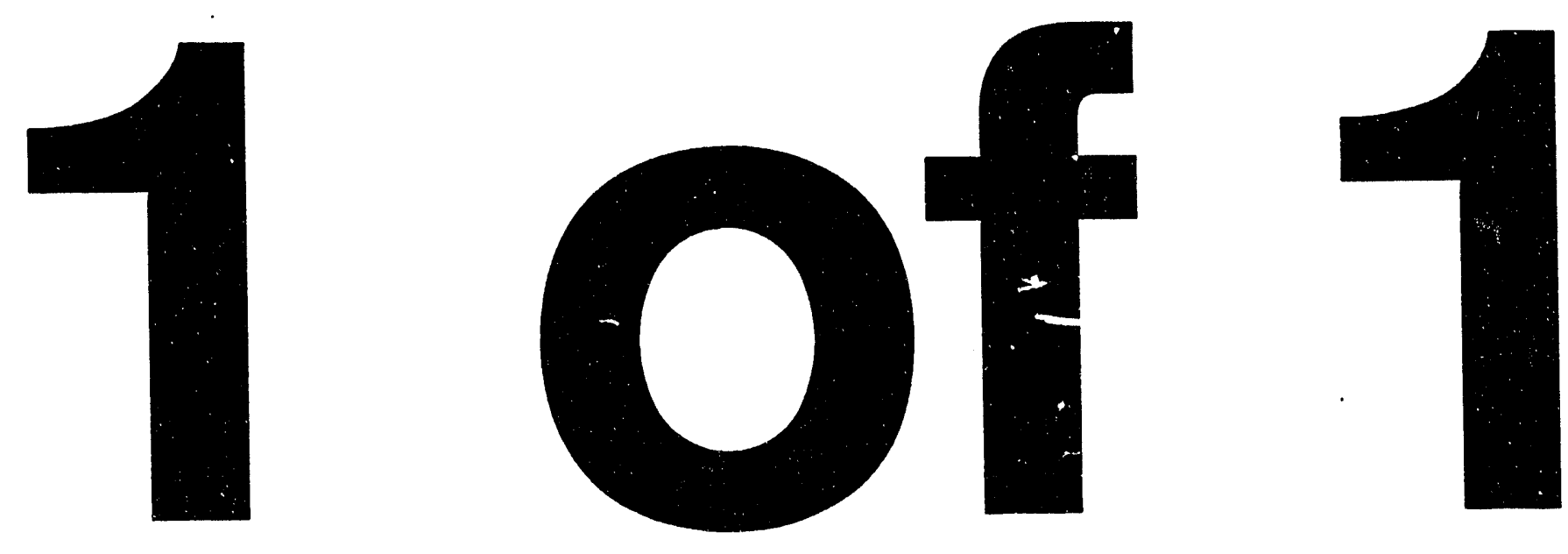


\title{
Dual-Band, Infrared Buried Mine Detection Using a Statistical Pattern Recognition Approach
}

\author{
Michael R. Buhl \\ Jose E. Hernandez \\ Gregory A. Clark \\ Sailes K. Sengupta
}

Engineering Research Division

Lawerence Livermore National Laboratory

Livermore, CA 


\section{ABSTRACT \\ Dual-Band, Infrared Buried Mine Detection Using a Statistical Pattern Recognition Approach}

The main objective of this work was to detect surrogate land mines, which were buried in clay and sand, using dual-band, infrared images. A statistical pattern recognition approach was used to achieve this objective. This approach is discussed and results of applying it to real images are given. 


\section{ACKNOWLEDGEMENTS}

We thank the members of the Airborne Standoff Mine Detection (ASMD) Project team at Lawrence Livermore National Laboratory, including the Automatic Target Recognition (ATR) Team: Robert J. Sherwood, for his work in acquiring, registering, and pre-processing the images, Nancy K. DelGrande, for her work in experimentation, sensors and data acquisition, Phil Durbin, for data acquisition, Marvin J. Barth, for project management, Paul C. Schaich, for algorithm development and image processing, Ronald J. Kane, for his work with ground penetrating radar and experimentation, John Feo, for parallel processing, David J. Fields, Program Leader, Michael Carter, Deputy Program Leader, and Arthur Toor, former Program Leader. 


\section{TABLE OF CONTENTS}

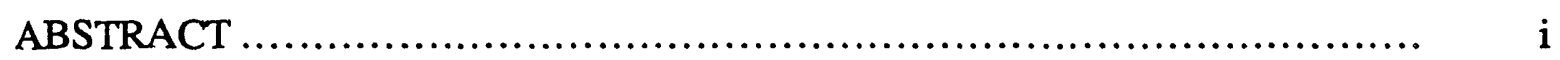

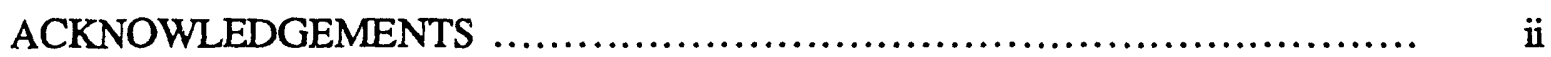

TABLE OF CONTENTS ..................................................... iii

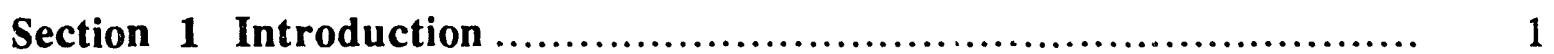

Section 2 Statistical Pattern Recognition Process........................ 2

2.1. Feature Selection ......................................... 2

2.2. Image Processing ....................................... 7

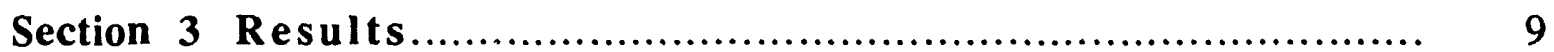

3.1. Data Set Description ........................................ 9

3.2. Feature Selection Results....................................... 10

3.2.1. Long and Short Wavelength Features ................. 12

3.2.2. Temperature and Emissivity Features ................. 13

3.3. Image Processing Results..................................... 15

3.3.1. Long and Short Wavelength Features ................. 15

3.3.2. Temperature and Emissivity Features ................... 17

Section 4 Conclusions .................................................... 19

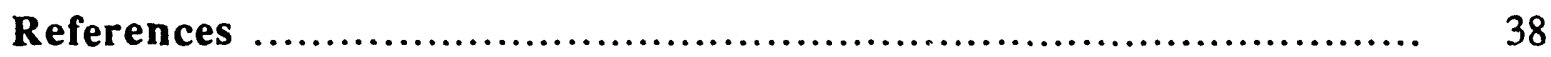




\section{Introduction}

The goal of this work was to detect and locate plastic and metal buried mines, given multiple registered images of regions of the earth. Past research hrs shown that it is extremely difficult to distinguish objects of interest from background clutter in images obtained from a single sensor. It is hypothesized, however, that information fused from a suite of sensors is likely to provide better detection reliability, because the suite of sensors detects a variety of physical properties that are more separable in feature space. Materials surrounding the objects of interest can include natural materials (soil, rocks, foliage, water, holes made by animals, and natural processes, etc.) and artifacts (objects made of metal, plastic and other materials). The sensor suite used for this study includes two infrared sensors (five micron and ten micron wavelengths). A statistical pattern recognition process used information from these sensors to detect elecromagnetic and thermal surrogate land mines, which are buried in clay and sand. This process uses advanced algorithms from the areas of automatic target recognition (ATR), computer vision, signal and image processing, and information fusion. The system used both physical principles and image processing for image interpretation [1-2].

This work is application research in progress. The individual algorithms used are advanced, but mostly known, and the novely of the work lies in the combination of the algorithms and their application to the very difficult and important problem of detecting buried land mines. To date, no successful operational system exists for airborne standoff detection of buried mines [1-2].

The remainder of this document is divided into three sections. Section two describes the statistical pattern recognition process. Section three presents the results of applying this process to real data. Lastly, conclusions based on the results of this work are discussed in section four. 


\section{Statistical Pattern Recognition Process}

The pattern recognition process consists of two main steps: the image processing step and the feature selection step. The image processing step classifies pixels of an image based upon first-order statistics, or features, which describe the distribution of intensity values within the neighborhood of the pixel. After all the pixels have been classified, additional processing is performed to isolate and smooth regions of mine pixels. Lastly, these regions are screened to determine which ones most likely represent mines. The feature selection process determines which subset of features when computed in the image processing step provide the greatest separability between the classes ("mines" and "no mines"). Since the feature selection process must be performed prior to the image processing, it will be presented first, in section 2.1. Following this, the image processing step will be presented in section 2.2 .

\subsection{Feature Selection}

The feature selection process is shown in Fig. 2.1.

The first step in feature selection is to pre-process the raw DBIR images. This step performs five main operations on these images. First, the geometrical distortions in the images are removed. These distortions result for various reasons, such as nonperpendicular orientation of the camera, sloping or rough terrain, and geometric aberrations of the lens. The processing in this step removes these distortions so that the images accurately represent the geometry of features on the surface [3]. Second, the long and short wavelength images are manually registered with respect to each other. After this step, corresponding pixels in the two images represent the same physical location. Third, these dual-band, registered images are used to construct two additional images which are related to the temperature and emissivity of objects in the scene [4-5]. This was done in an attempt to determine whether images which represent temperature and emissivity result in higher 


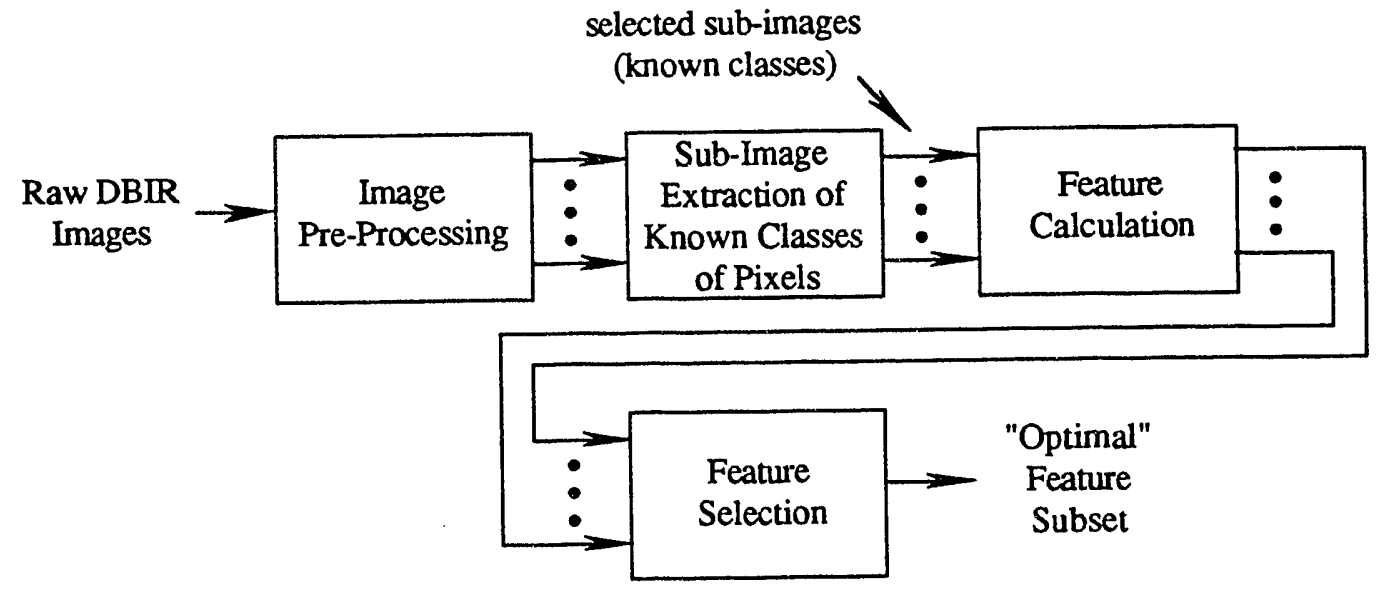

Fig. 2.1. Feature selection process

detection probabilities than can be achieved using images which simply represent the infrared intensities. Since the temperature and emissivity of objects are related to the long and short wavelength intensity values, it is possible to decouple these quantities using the following equations,

$$
\begin{aligned}
& \text { Temperature Image }(\ln \mathrm{T})=\ln \frac{(\mathrm{S} / \mathrm{s})}{(\mathrm{L} / \mathrm{l})} \text { and } \\
& \text { Emissivity Image }(\ln \mathrm{E}) \quad=\ln \frac{(\mathrm{S} / \mathrm{s})}{(2 \mathrm{~L} / \mathrm{l})},
\end{aligned}
$$

where $S$ is the short wavelength ( 5 microns) intensity image, $s$ is the average value of the pixels in $\mathrm{S}, \mathrm{L}$ is the long wavelength (10 microns) intensity image, and $\mathrm{l}$ is the average value of the pixels in $\mathrm{L}$. Fourth, all four inages are normalized with respect to their background. This is accomplished by subtracting the mean of the background from the images and dividing this result by the standard deviation of the background. This is done so that the distribution of intensity values are consistent relative to the background in all the images $[1,2,6]$.

The next step shown in Fig. 2.1 is to extract sub-images which represent classes of pixels from the pre-processed images. These sub-images are used to define the neighborhood of a pixel from which the features are to be computed. These features, often 
referred to as a feature vector, are statistical measures which describe various probabilistic intensity distributions within the sub-image. Two commonly used classes of features are amplitude features and textural features. Amplitude features describe the distribution of intensity values within the neighborhood (sub-image) of a pixel [7]. In contrast, textural features describe the distribution of the sum and difference of intensity values within the neighborhood of a pixel. These textural distributions are given for some prescribed spatial relationship among two pixels [8]. For the results given in this report, only amplitude features were used. This was done fcr a couple reasons. First, the amplitude features are less dependent upon the pixel resolution than are textural features, and therefore the results are more gereral. Second, the number of pixels in the sub-images is only marginally sufficient for using texture features. The amplitude features which were generated are given in Table 2.1 .

\begin{tabular}{|c||c|}
\hline $\begin{array}{c}\text { Feature } \\
\text { Name }\end{array}$ & $\begin{array}{c}\text { Mathematical } \\
\text { Representation }\end{array}$ \\
\hline Mean & $\mu=\sum \mathrm{x} \mathrm{P}(\mathrm{x})$ \\
\hline Standard Deviation & $\sigma=\left[\sum(\mathrm{x}-\mu)^{2} \mathrm{P}(\mathrm{x})\right]^{0.5}$ \\
\hline Skewness & $\frac{1}{\sigma^{3} \sum(\mathrm{x}-\mu)^{3} \mathrm{P}(\mathrm{x})}$ \\
\hline Kurtosis & {$\left[\frac{1}{\sigma^{4}} \sum(\mathrm{x}-\mu)^{4} \mathrm{P}(\mathrm{x})\right]-3$} \\
\hline Energy & $\sum[\mathrm{P}(\mathrm{x})]^{2}$ \\
\hline Entropy & $-\sum \mathrm{P}(\mathrm{x}) \log _{2}[\mathrm{P}(\mathrm{x})]$ \\
\hline
\end{tabular}

Notes:

1.) $P(x)$ is the probability density function of intensity values.

2.) All summations are over all intensity values.

Table 2.1. Amplitude features 
After the features are computed, the last step is to perform the actual feature selection on these vectors. This step determines which subset of features should result in the best classification. This is typically accomplished by computing a distance measure which is the sum of probabilistic distances between all pair-wise combinations of classes [9]. The feature subset which results in the greatest distance measure will tend to provide the greatest separability between classes. Therefore, the feature selection process attemipts to select the subset of features which results in the greatest class separability, hence the best classification accuracy.

The feature selection block of Fig. $2.1 \mathrm{can}$ be broken into two parts: the feature subset search algorithm and the separability disiance computation. The feature subset search algorithm determines the sequence of feature subsets which are tested, and the separability distance determines how the probabilistic distance is computed for a given subset. It should be noted that these parts are by no means completely independent of each other, for the separability distance computed at one step effects the feature subset tested at the next step. Commonly used feature subset search algorithms are the branch and bound, sequential forward selection, and sequential backward selection algorithms, while typical separability distances are the Bhattacharyya, Chernoff, Matusita, Mahalanobis, and divergence distances [9]. In order to compute the separability distance accurately, large data sets must be available. Unfortunately, for the data and results presented in this paper this was not the case. Therefore an alternate measure of class separability had to be devised.

Since the ultimate goal of this work was to detect mines, the probability of detection (using a given classifier) was used as the separability distance. Next, a feature subset search algorithm had to be chosen. The branch and bound algorithm requires that the criterion measure be monotonic in the following sense: given a feature subset, adding one more feature to this set will result in a greater criterion. Unfortunately, the probability of 
detection criterion does not satisfy monotonicity because adding features to a subset may "confuse" the classifier, resulting in a lower probability of detection. As a result, the branch and bound algorithm could not be used. The other feature subset search algorithms are sub-optimal in the sense that they do not always select the subset of features which result in the largest separability distance. Therefore a "brute-force" method of testing all possible feature combinations was used. Since the separability distance was computed for all combinations of features, this approach was optimal. This process is shown in Fig. 2.2 .

The classifier shown in this figure accepts the subset of features and classifies them based on some prescribed method. A variety of classifiers exist and can be implemented. Some commonly used classifiers are the nearest neighbor classifier and neural network classifiers. The nearest neighbor classifier was used for this problem because it is relatively simple, it performs well, and there is no need to manually adjust tuning parameters as is the case for some other classifiers, including neural networks. This method computes the Euclidean distances between a feature vector which is to be classified and a set of feature vectors whose classes are known (training data). The feature vector

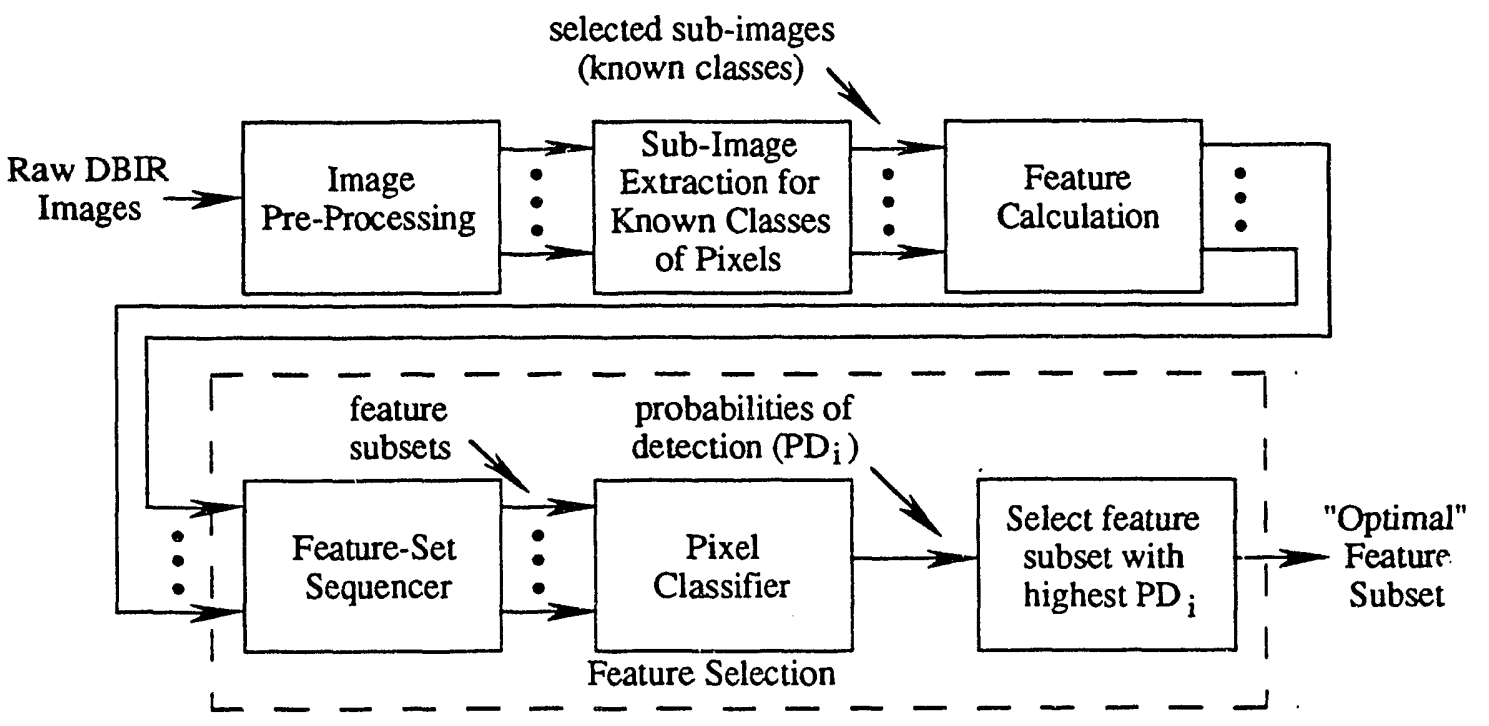

Fig. 2.2. Feature selection process which was used for mine detection 
being tested is assumed to belong to the same class as the feature vector which produces the smallest distance [10]. Since the training set (which will be described in section 3 ) is small, the "hold one out" technique was used to compute the probability of detection for each feature subset. This technique uses all the known feature vectors for the classifier except for one, which is used as input to test the classifier. Therefore, the classifier must be trained once for every feature vector which is to be classified.

After feature selection has been performerl, the "optimal" feature subset is used to perform the image processing. This is presented in the next section.

\subsection{Image Processing}

A block diagram of the image processing is shown in Fig. 2.3. The first step shown in this figure is to pre-process the raw DBIR images. This step performs the same operations as those discussed in section 2.1 , and therefore will not be discussed here. After the pre-processing, a known data base must be created from which the pixel classification will be based. This is accomplished by first extracting sub-images of known classes from the pre-processed images. Following this, the "optimal" subset of features which were determined in the feature selection are computed for each sub-image. The resulting feature vectors will provide the training data for the pixel classifier.

Next, all the pixels in the pre-processed image must be classified as a "mine" or a "no mine" pixel. This is accomplished by extracting a sub-image centered around each pixel in the images, and computing the "optimal" subset of features for this sut-image. The next step is to use a pixel classifier to determine which class the sub-image represents at the center pixel. The classifier which was used was the nearest neighbor classifier described in section 2.1 .

The output of the pixel classifier is a binary image in which ones represent possible "mine" pixels and zeros represent "no mine" pixels. These images will be referred to as 


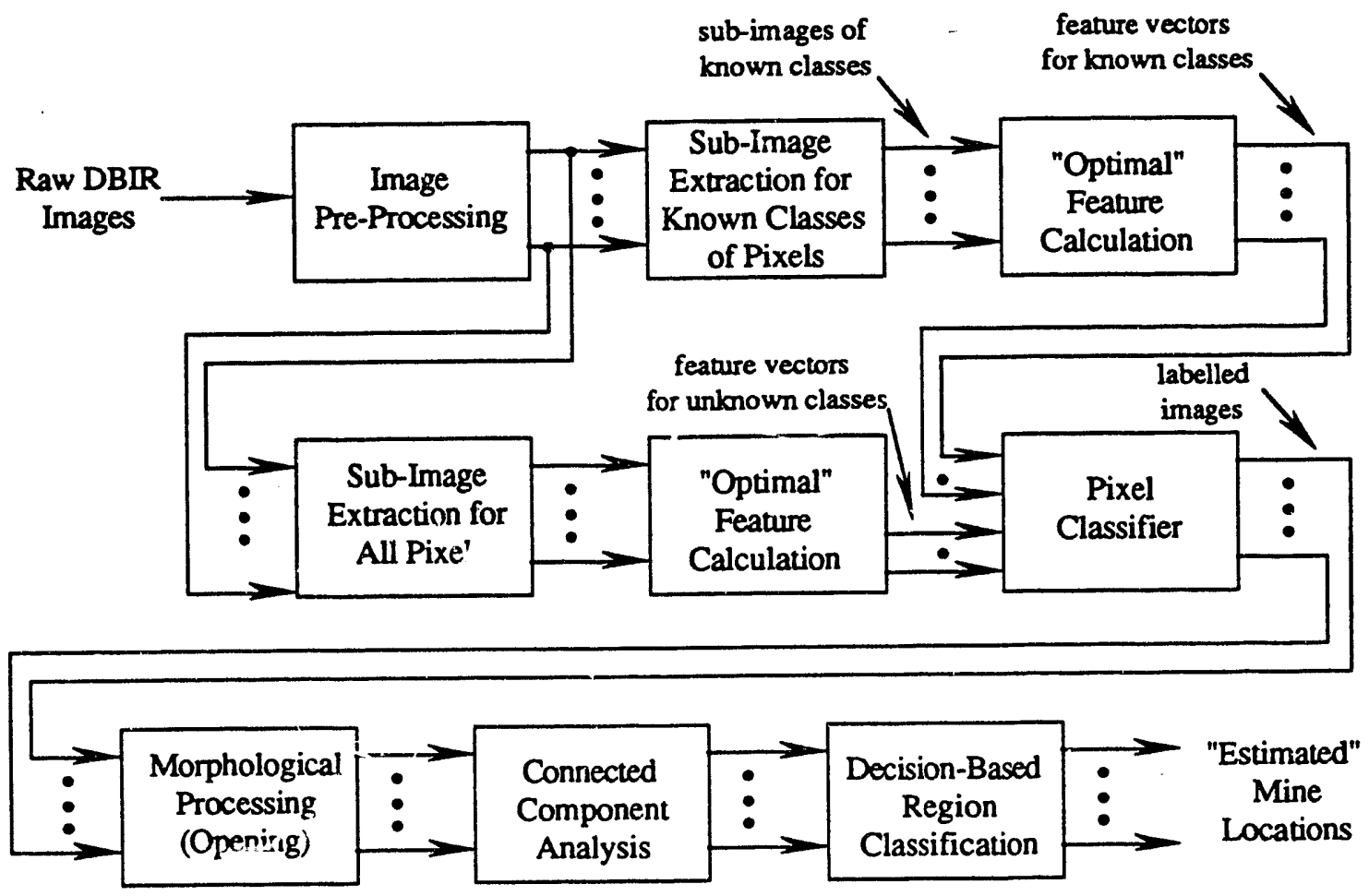

Fig. 2.3. Image Processing

labelled images. Labelled images often consist of distinct regions which are connected via narrow channels. Additionally, these regions may have sharp edges or caps. In order to smooth these regions and eliminate the narrow channels, a morphological opening operation is performed on the image [11]. This operation involves two steps. First, the binary image is "eroded". Erosion marks, or sets to one, all the pixels which when logically "anded" with a structuring element (region of ones) produce all ones. Following this operation, the "eroded" image is "dilated". Dilation marks all the pixels which when logically "anded" with the same structuring element produce at least a single one.

The next step is to connect all the "mine" pixels into regions. This is accomplished with connected component analysis [12]. Using this analysis, a region is defined as a group of pixels connected to each other via traversal in either eight adjacent directions (north, northeast, east, southeast, south, southwest, west, and northwest) or four adjacent directions (north, east, south, west). Regions formed using the eight direction traversals 
are referred to as 8-connected regions, and regions formed using the four direction traversals are referred to as 4-cornected regions. For the results given in this report, 4connected regions were formed.

After all the "mine" pixels have been combined into regions, the next step is to determine which regions are likely to represent mines. This region classification is based solely upon the region size. Small regions which typically represented clutter are eliminated from consideration because of their small size. The remaining regions, which contain at least a specifiec' number of pixels, are assumed to be mine regions.

The next section describes the results of applying the steps discussed in sections 2.1 and 2.2 to real data.

\section{Results}

As previously stated, the detection of mines is a two step process. First, the features which result in the best classification are determined from feature vectors of known classes (feature selection). Following this, images are processed to determine where mines may be buried (image processing). This section describes the results obtained when applying these steps to real data.

Section 3.1 describes the data which was used to generate these results, section 3.2 presents the results obtained in the feature selection step, and section 3.3 presents the results obtained in the image processing step.

\subsection{Data Set Description}

The data which were used for feature selection and image processing were taken from four surrogate mine pits $(1,2 a, 2 b, 2 c)$ located on site at Lawrence Livermore National Laboratory. Three of the pits consisted of clay terrain and one consisted of sand terrain. Additionally, images were acquired at daytime and nighttime to determine whether the time 
of day affected the probability of detecting mines. The terrain and the number of daytime and nighttime images for each pit are given in Table 3.1. These pits contained electromagnetic and thermal surrogate land mines. Because the surrogate mines cannot simultaneously simulate both the dielectric and thermal properties of high explosives, separate sets of thermal and dielectric surrogate mines were used. In addition to buried mines, the pits also contained filled-in holes, which were used to simulate buried mines. This could be done because experiments showed that the thermal signatures of filled holes closely approximate the thermal signatures of buried mines.

\begin{tabular}{|c||c|c|c|}
\hline $\begin{array}{c}\text { Pit } \\
\text { Number }\end{array}$ & Terrain & $\begin{array}{c}\text { Number of } \\
\text { Daytime } \\
\text { Images }\end{array}$ & $\begin{array}{c}\text { Number of } \\
\text { Nighttim? } \\
\text { Images }\end{array}$ \\
\hline \hline 1 & clay & 1 & 2 \\
\hline $2 \mathrm{a}$ & clay & 1 & 1 \\
\hline $2 \mathrm{~b}$ & sand & 2 & 2 \\
\hline $2 \mathrm{c}$ & clay & 2 & 1 \\
\hline
\end{tabular}

Table 3.1. Terrain and number of images for mine pits

\subsection{Feature Selection Results}

In order to perform the feature selection, a set of feature vectors of known classes had to be generated. These feature vectors were generated by manually selecting known square regions within the images and performing a feature calculation on these sub-images. All the mines were combined into one class because their infrared signatures were very similar. Additionally, the filled-in holes were combined with the buried mines because some preliminary results indicated that it is very difficult to differentiate them using thermal IR. Moreover, it is safe to assume, in a typical operational situation, that wherever a filled-in hole exists, a mine may also exist. Therefore, the first class which will be referred to as the "riine" class consisted of metal mines, plastic mines, and filled-in holes. The second class 
will be referred to as the "no mine" class. This class consisted of background, clutter, and fiducials. Clutter was defined as regions in the image which were in the background but had intensity values similar to those in the "mine" class. The number of sub-images which were taken from each pit are given in Table 3.2.

Because daytime and nighttime images have differing thermal characteristics, they were evaluated separately. Additionally, the clay and the sand images had differing thermal characteristics, so they were also evaluated separately. In an attempt to determine whether features which represent temperature and emissivity characteristics result in better detection probabilities than features which simply represent long and short wavelength intensity values, the four cases given above were repeated once for long and short wave-length features and once for temperature and emissivity features. Therefore, the feature selcction process, shown in Fig. 2.2, was performed for eight cases: daytime, clay, short and lorig; nighttime, clay, short and long; daytime, sand, short and long; nighttime, sand, short and long; daytime, clay, temperature and emissivity; nighttime, clay, temperature and emissivity; daytime, sand, temperature and emissivity; and nighttime, sand, temperature and emissivity.

\begin{tabular}{|c||c|c|c|c|c|}
\hline \multirow{2}{*}{\begin{tabular}{c}
\multirow{2}{*}{$\begin{array}{c}\text { Pit } \\
\text { Number }\end{array}$} \\
\cline { 2 - 6 }
\end{tabular}} & $\begin{array}{c}\text { Buried } \\
\text { Mines }\end{array}$ & $\begin{array}{c}\text { Filled } \\
\text { Holes }\end{array}$ & Fiducials & $\begin{array}{c}\text { Back- } \\
\text { ground }\end{array}$ & Clutter \\
\hline \hline 1 & 0 & 3 & 8 & 12 & 5 \\
\hline $2 \mathrm{a}$ & 6 & 3 & 16 & 7 & 3 \\
\hline $2 \mathrm{~b}$ & 9 & 3 & 16 & 9 & 3 \\
\hline $2 \mathrm{c}$ & 3 & 3 & 16 & 9 & 6 \\
\hline
\end{tabular}

Table 3.2. Number of samples for each class 
For each of these cases, features were computed for sub-images of size 21 pixels by 21 pixels. This size was chosen because this was approximately the largest sub-image which could be completely contained inside the boundary of a mine. Since six amplitude features were computed for each sub-image, each feature vector could contain up to twelve features. This resulted in $4095\left(2^{12}-1\right)$ possible feature subsets. For each of these subsets, the probability of detection was computed using the "hold one out" technique. The following two sections present the results of the feature selection process when applied to the long and short wavelength features and the temperature and emissivity features, respectively.

\subsubsection{Long and Short Wavelength Features}

The feature subsets which resulted in the highest probability of "mine" detection using the long and short wavelength features are given in Table 3.3. The accuracy of classification is also given in these tables. The probability of detection is the percentage of "mine" samples classified correctly, and the probability of false alarm is the percentage of "no mine" samples classified as "mines". Beneath the probabilites of detection in the table are shown the ratios of the number of "mines" correctly classified to the number of actual "mines" tested. Similarily, beneath the probabilities of false alarm are shown the ratios of the number of "no mine" samples classified as "mines" to the total number of "no mine" samples tested.

A few points should be noted regarding these results. First, when the terrain is clay, the probabilities of detection are high and the probabilities of false alarm low. In fact, for the nighttime images perfect results were achieved. Moreover, these results were obtained with relatively few features. For the nighttime images, the "optimal" feature subset consisted of only one short wavelength feature. For the sand terrain images, the probabilities of detection are again high. However, the probabilities of false alarm are higher than those obtained for the clay terrain images. 


\begin{tabular}{|c|c|c|c|}
\hline Time of Day/Terrain & $\begin{array}{c}\text { "Optimal" } \\
\text { Feature Subset }\end{array}$ & $\begin{array}{l}\text { Probability of } \\
\text { Detection }\end{array}$ & $\begin{array}{l}\text { Probability of } \\
\text { False Alarm }\end{array}$ \\
\hline Daytime, Clay & $\begin{array}{c}\text { mean (long) } \\
\text { standard deviation (long) } \\
\text { skewness (long) }\end{array}$ & $\begin{array}{l}95.83 \\
(23 / 24)\end{array}$ & $\begin{array}{c}0.80 \\
(1 / 113)\end{array}$ \\
\hline Nighttime, Clay & mean (short) & $\begin{array}{l}100.0 \\
(21 / 21)\end{array}$ & $\begin{array}{l}0.00 \\
(0 / 107)\end{array}$ \\
\hline Daytime, Sand & $\begin{array}{l}\text { standard deviation (long) } \\
\text { kurtosis (long) } \\
\text { standard deviation (short) } \\
\text { skewness (short) }\end{array}$ & $\begin{array}{l}87.50 \\
(21 / 24)\end{array}$ & $\begin{array}{r}12.50 \\
(7 / 56)\end{array}$ \\
\hline Nighttime, Sand & $\begin{array}{c}\text { standard deviation (long) } \\
\text { mean (short) }\end{array}$ & $\begin{array}{l}100.0 \\
(24 / 24)\end{array}$ & $\begin{array}{l}5.36 \\
(3 / 56)\end{array}$ \\
\hline
\end{tabular}

Table 3.3. Feature selection results for long and short wavelength features

\subsubsection{Temperature and Emissivity Features}

The results of the feature selection process using the temperature and emissivity features are given in Table 3.4. For the clay terrain images, the results are very similar to those given in Table 3.3. The only significant difference appears to be the number of features in the "optimal" subset. The "optimal" subset for the long and short wavelength images consist of three features for daytime and one feature for nighttime, whereas for the temperature and emissivity images the "optimal" subset consisted of four features for daytime and two features for nighttime. However, these results are still encouraging since relatively few features are required to achieve the best classification. For the sand terrain, the results are also very similar to those in Table 3.3. When comparing the results for the sand terrain images to those for the clay terrain images, it is evident that for sand terrain the 


\begin{tabular}{|c|c|c|c|}
\hline Time of Day/Terrain & $\begin{array}{l}\text { "Optimal" } \\
\text { Feature Subset }\end{array}$ & $\begin{array}{l}\text { Probability of } \\
\text { Detection }\end{array}$ & $\begin{array}{l}\text { Probability of } \\
\text { False Alarm }\end{array}$ \\
\hline Daytime, Clay & $\begin{array}{c}\text { mean }(\ln T) \\
\text { kurtosis }(\ln T) \\
\text { skewness }(\ln E) \\
\text { entropy }(\ln E)\end{array}$ & $\begin{array}{l}95.83 \\
(23 / 24)\end{array}$ & $\begin{array}{c}1.77 \\
(2 / 113)\end{array}$ \\
\hline Nighttime, Clay & $\begin{array}{c}\text { mean }(\ln T) \\
\text { standard deviation }(\ln T)\end{array}$ & $\begin{array}{l}100.0 \\
(21 / 21)\end{array}$ & $\begin{array}{l}0.00 \\
(0 / 107)\end{array}$ \\
\hline Daytime, Sand & $\begin{array}{c}\text { mean }(\ln \mathrm{T}) \\
\text { standard deviation }(\ln \mathrm{T}) \\
\text { mean }(\ln \mathrm{E}) \\
\text { standard deviation }(\ln \mathrm{E})\end{array}$ & $\begin{array}{l}83.33 \\
(20 / 24)\end{array}$ & $\begin{array}{l}10.71 \\
(6 / 56)\end{array}$ \\
\hline Nighttime, Sand & $\begin{array}{c}\text { mean }(\ln T) \\
\text { standard deviation }(\ln T) \\
\text { skewness }(\ln T) \\
\text { skewness }(\ln E) \\
\text { kurtosis }(\ln E)\end{array}$ & $\begin{array}{l}87.50 \\
(21 / 24)\end{array}$ & $\begin{array}{c}10.71 \\
(6 / 56)\end{array}$ \\
\hline
\end{tabular}

Table 3.4. Feature selection results for temperature and emissivity features

probabilities of detection are lower and the probabilities of false alarm higher. This result is not surprising because visual inspection of these images indicate that the "mines" and "no mines" classes appear more different (separable) in the clay terrain images than in the sand terrain images.

Although the results in Tables 3.3 and 3.4 are very encouraging, it must be remembered that they were generated based on a small data set. Additionally, they were generated using feature vectors which lay distinctly in one class or the other. This is hardly the case when processing unknown images. In order to determine results for this case, the classification was run over all the pixels in a given image (image processing). These results are given in the next section. 


\subsection{Image Processing Results}

For this step, a feature vecior was generated for every pixel in an image (sub-image surrounding the pixel) and classified. These feature vectors were computed from subimages of 21 by 21 pixels. This size was chosen since it was approximately the largest sub-image which could be completely contained inside the boundary of a mine. The results of this pixel classification process were labelled images. These images were then processed with a morphological opening operator. After observing the labelled images, it was decided that a structuring element of 5 by 5 pixels would be large enough to close the narrow channels and smooth major regions. After this operation, connected component analysis was performed on these smoothed images to form 4-connected regions. Lastly, regions which contained fewer than 200 pixels were eliminated from consideration as possible mine regions. This number was chosen because it was judged that regions with fewer than 200 pixels were much smaller than mines and most likely a result of clutter. An actual mine would consist of about 800 pixels since they are about 32 pixels in diameter. Lastly, the centroids of the remaining regions were computed, and assumed to be the estimated mine locations. The following two sections present the results of the image processing when applied to the images using long and short wavelength features and the temperature and emissivity features.

\subsubsection{Long and Short Wavelength Features}

The images produced using only the long and short wavelength "optimal" features (Table 3.3) are shown in Fig's 3.1 to 3.8. In these figures, the long and short wavelength IR images are shown along with two additional images, which are described below. For each case, one set of images for each pit is shown. Fig's 3.1 to 3.3 show the results obtained for the daytime, clay terrain case. Likewise, Fig's 3.4 to 3.6 show the results obtained for the nighttime, clay terrain case. Fig. 3.7 shows the results obtained for the daytime, sand terrain case. Finally, Fig. 3.8 shows the results obtained for the nighttime, 
sand cerrain case. In the long and short wavelength images, the square outlines represent 21 by 21 pixel sub-images centered on the "mines". The other noticeable round areas which appear in some of these images at regular intervals are fiducials which were used in the image pre-processing. The labelled images are the resulting images after pixel classification (see Fig. 2.3). The white regions indicate pixels classified as "mines". The images in the lower right corner are the result of the image processing step. In these images, the grey circles with the black cross-hair indicate "actual" mine locations. These circles have a radius of 16 pixels, which is the approximate size of a mine. The small white circles in these images indicate "estimated" mine locations. For the following results, it will be assumed that if the estimated mine location circle (white) and the actual mine circle (grey) intersect, the actual mine was successfully detected. Given this specification, the probability of detection and false alarm computed from these images using only long and short wavelength features are given in Table 3.5. The probability of detection is calculated as the ratio of the number of actual mine location circles for which the actual mine location and an estimated mine location intersect to the number of actual mines tested. The probability of false alarm is calculated as the number of estimated mine location circles which do not intersect an actual mine location circle to the total number of estimated mine location circles.

\begin{tabular}{|c||c|c|}
\hline Time of Day/Terrain & $\begin{array}{c}\text { Probability of } \\
\text { Detection }\end{array}$ & $\begin{array}{c}\text { Probability of } \\
\text { False Alarm }\end{array}$ \\
\hline \hline Daytime, Clay & $\begin{array}{c}100.0 \\
(18 / 18)\end{array}$ & $\begin{array}{l}0.00 \\
(0 / 18)\end{array}$ \\
\hline Nighttime, Clay & $\begin{array}{c}88.89 \\
(16 / 18)\end{array}$ & $\begin{array}{l}0.00 \\
(0 / 16)\end{array}$ \\
\hline Daytime, Sand & $\begin{array}{c}83.33 \\
(10 / 12)\end{array}$ & $\begin{array}{l}47.06 \\
(8 / 17)\end{array}$ \\
\hline Nighttime, Sand & 75.00 & 23.08 \\
$(9 / 12)$ & $(3 / 13)$ \\
\hline
\end{tabular}

Table 3.5. Image processing results for long and short wavelength features 
These results appear very good for the clay terrain cases. For the daytime images, all the mines were detected and there were no false alarms. The probability of detection went down slightly at nighttime, however the probability of false alarm was still zero. The two mines from pit $2 \mathrm{a}$ (Fig. 3.5) which were not detected were the result of the size constraint put on the minimum number of pixels in a mine region. The region in the lower left corner of the labelled image contained 67 pixels after the morphological processing, and the region in the lower right corner contained 95 pixels after the morphological processing. In order to correctly detect these regions, the size constraint could be lowered. However, lowering this value will increase the probability of false alarm.

For the sand terrain images, the results were not as encouraging. Both the daytime and nighttime images resulted in lower probabilities of detection and higher probabilities of false alarm than either of the clay terrain cases. This was not surprising since the thermal properties of the "mine" and "no mine" classes are very similar from visual inspection. This similarity is evident directly from these images. It is interesting to note that the nighttime labelled image contains square outlines of "mine" pixels. This is an effect of the fiducials. When a sub-image contains a small part of a fiducial, the standard deviation of the long wavelength sub-image is increased enough and the mean of the short wavelength sub-image is decreased enough to make these pixels appear as "mine" pixels. Fortunately, the morphological opening eliminates these square outlines from further consideration as mine regions.

\subsubsection{Temperature and Emissivity Features}

The images produced using only the temperature and emissivity features (Table 3.4) are shown in Fig's 3.9 to 3.16. Fig's 3.9 to 3.11 show the results obtained for the daytime, clay terrain case. Likewise, Fig's 3.12 to 3.14 show the results obtained for the nighttime, clay terrain case. Fig. 3.15 shows the results obtained for the daytime, sand terrain case. Finally, Fig. 3.16 shows the results obtained for the nighttime, sand terrain 
case. In these images, the square outlines represent 21 by 21 pixel sub-images centered on the "mines". The other noticeable round areas which appear in some of these images at regular intervals are fiducials. For Fig's 3.9 to 3.16, the images in the lower left and right corners are the labelled images and the resulting images of the processing, respectively. Again, the grey circles in the resulting images are the actual mine locations, and the white circles are the estimated mine locations. It is assumed that if these circles intersect, the mine was detected correctly. Given this specification, the probabilities of detection and false alarm computed from these images using only temperature and emissivity features are given in Table 3.6. The probability of detection is calculated as the ratio of the number of actual mine location circles for which the actual mine location and an estimated mine location intersect to the number of actual mines tested. The probability of false alarm is calculated as the number of estimated mine location circles which do not intersect an actual mine location circle to the total number of estimated mine location circles.

Again, these results appear very good for the clay terrain cases. For the daytime images, the probability of detection is slightly lower than that obtained using only the short and long wavelength features. However, there were again no false alarms. For the nighttime case, the probability of detection was slightly higher than in the daytime, and

\begin{tabular}{|c||c|c|}
\hline Time of Day/Terrain & $\begin{array}{c}\text { Probability of } \\
\text { Detection }\end{array}$ & $\begin{array}{c}\text { Probability of } \\
\text { False Alarm }\end{array}$ \\
\hline Daytime, Clay & $\begin{array}{l}83.33 \\
(15 / 18)\end{array}$ & $\begin{array}{l}0.00 \\
(0 / 15)\end{array}$ \\
\hline Nighttime, Clay & $\begin{array}{l}94.44 \\
(17 / 18)\end{array}$ & $\begin{array}{l}0.00 \\
(0 / 17)\end{array}$ \\
\hline Daytime, Sand & $\begin{array}{c}66.67 \\
(8 / 12)\end{array}$ & $\begin{array}{l}42.86 \\
(6 / 14)\end{array}$ \\
\hline Nighttime, Sand & $\begin{array}{l}91.67 \\
(11 / 12)\end{array}$ & $\begin{array}{l}7.64 \\
(1 / 12)\end{array}$ \\
\hline
\end{tabular}

Table 3.6. Image processing results for temperature and emissivity features 
there were no false alarms. The mines which were not detected were the result of the size constraint put on the minimum number of pixels in a mine region. These regions, could have been detected correctly by lowering this threshold. However, doing so could increase the number of false alarms.

For the daytime sand terrain images, the results were not as encouraging. These images resulted in lower probabilities of detection and higher probabilities of false alarm than either of the clay terrain cases. This was not surprising since the thermal properties of the "mine" and "no mine" classes are very similar. This similarity is evident directly from these images. The daytime images contain square outlines of "mine" pixels similar to those which appeared in the nighttime, sand results for the long and short wavelength features. This again is due to the fiducials. When the sub-image contains a small part of a fiducial, the feature vector becomes biased toward the "mines" class. This results in these regions being labeled as "mines". The morphological opening eliminates these square outlines from further consideration as mine regions. The results for the nighttime images were surprisingly good. The probability of detection was higher than any of the other three sand terrain cases. Additionally, the probability of false alarm was substantially lower.

\section{Conclusions}

Several conclusions can be drawn based on the results of this work.

- In general, it is easier to detect "mines" buried in clay than it is to detect "mines" buried in sand.

This result was not surprising because visual inspection of the images indicated that the "mines" class was more noticeable in the clay terrain images than in the sand terrain images. For the clay terrain images, it was difficult to differentiate between filled-in holes and buried mines. This confirms our knowledge from physical principles that the disturbed earth plays an important role in the mine detection. The difference between the mine 
detection in clay terrain to that in the sand terrain may be due mostly to the thermal properties of the disturbed soil [4-6]. The thermal properties of a filled-in sand hole tend to be more similar to undisturbed sand than the filled-in clay hole is to undisturbed clay. This also indicates that the mine detection may be dependent upon the length of time between the burying of the mines and the acquisition of the images [4-6]. For this study the data were acquired within several months of burial. Before moving on the the next point, it should be noted that although the results tended to be better when the mines were buried in clay terrain, the nighttime sand terrain images when processed with temperature and emissivity features provided encouraging results (probability of detection $=91.67 \%$ and probability of false alarm $=7.64 \%$ ).

- In general, there does not appear to be any clear advantage to using nighttime images over daytime images when detecting mines.

When comparing the results after the pixel classification step (labelled images), the daytime images appear to have more thermal clutter than the nighttime images [5]. Fortunately, the morphological opening along with the region size constraint tends to eliminate these small mine regions. The result is classification accuracies which are not significantly different for both daytime and nighttime images. When using long and short wavelength features with the clay terrain, the daytime results are better than the nighttime results (see Table 3.5). The probability of detection is higher and the probability of false alarm is identical to that obtained from nighttime images. For the sand terrain, the results are mixed. The daytime images resulted in a higher probability of detection than the nighttime images, but the probability of false alarm was also higher. When using the temperature and emissivity features with the clay terrain, the nighttime results are better than the daytime results. The probability of detection is higher and the probability of false alarm is identical to that obtained from daytime images. For the sand terrain, the nighttime results are again better than the daytime results. The probability of detection is higher and 
the probability of false alarm lower than the daytime results. Since these results tend to be mixed, it is unjustified to state that one set of images (daytime or nighttime) yield clearly better results than the other set.

- There seems to be little difference between results acquired using amplitude features of the long and short wavelength images and results acquired using amplitude features of the temperature and emissivity images.

This result should not imply that the temperature and emissivity images do not yield any additional information, and therefore should not be computed. In fact, previous research has shown that emissivity features are able to differentiate between surface clutter and buried object very well [5]. However, for this study no surface objects were present so this technique could not be adequately exercised. Additionally, man-made surface objects of different materials may appear very similar in the long and short wavelength images. However in the emissivity images, these objects may appeai very different. Therefore, if the images consist of much surface clutter, or if it is of interest to detect objects of different man-made materials, the emissivity features may be very important.

- For some cases, the best features only relied on one band of IR.

This results occurreci for the daytime, clay, short and long wavelength case and the nighttime, clay, short and long.wavelength case. Since the two band of IR provide more information than one band, it was expected that by using both bands higher probabilities of detection and lower probabilites of false alarm would result. However, for the results given in this report, the images contained little surface clutter, and the detection of mines was a relatively simple. Under more realistic conditions, it is still expected that sensor fusion will aid in the detection of mines. 


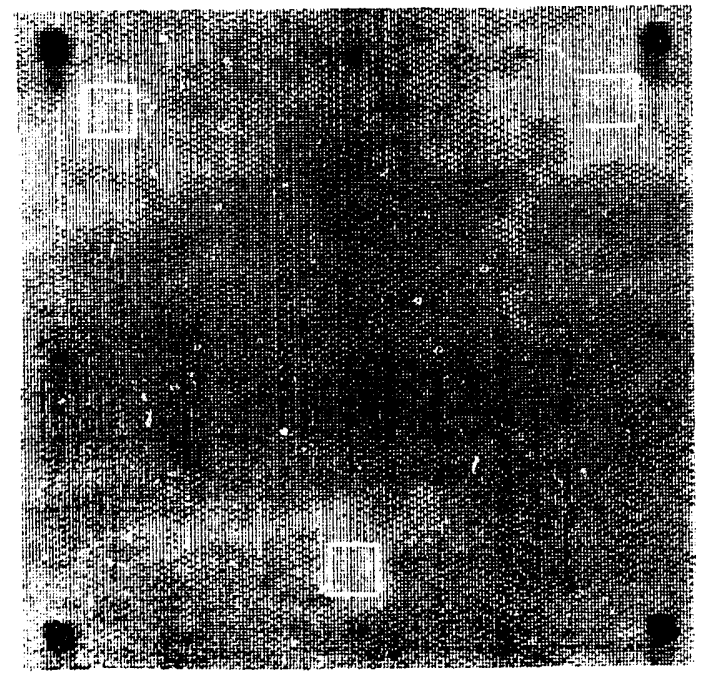

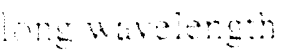

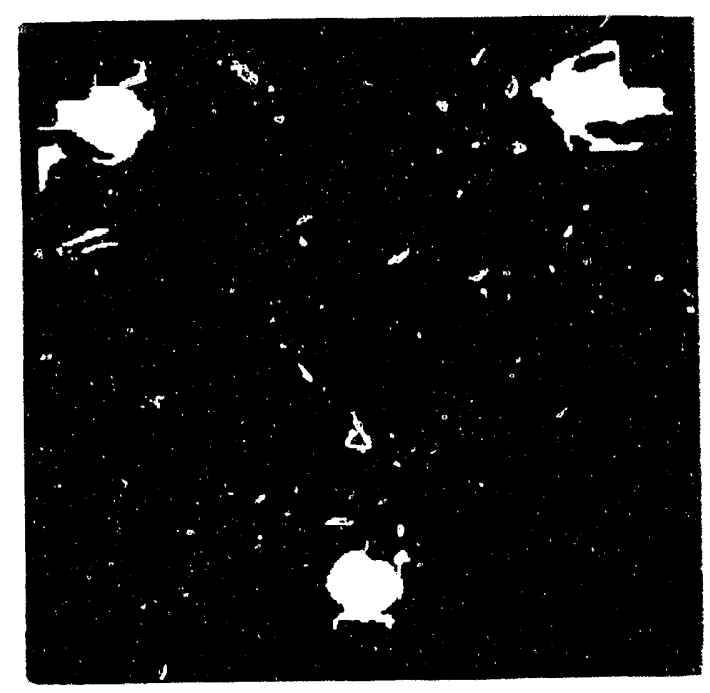

$\therefore \cdots \cdots \cdots$

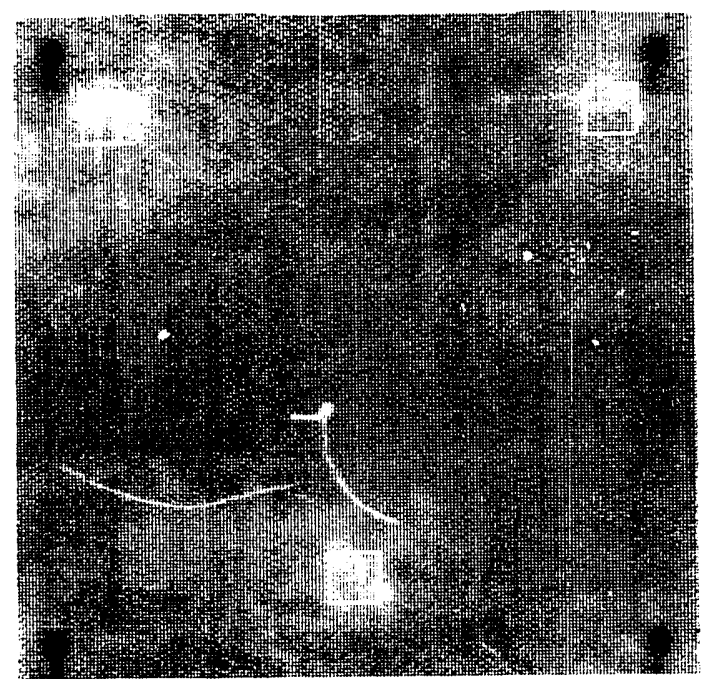

Sno sanelength

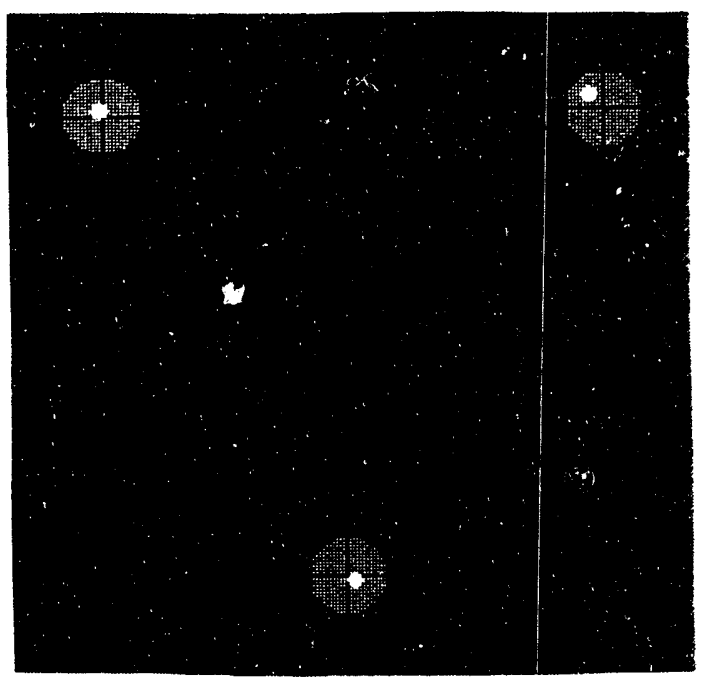

$\because \cdots$ 

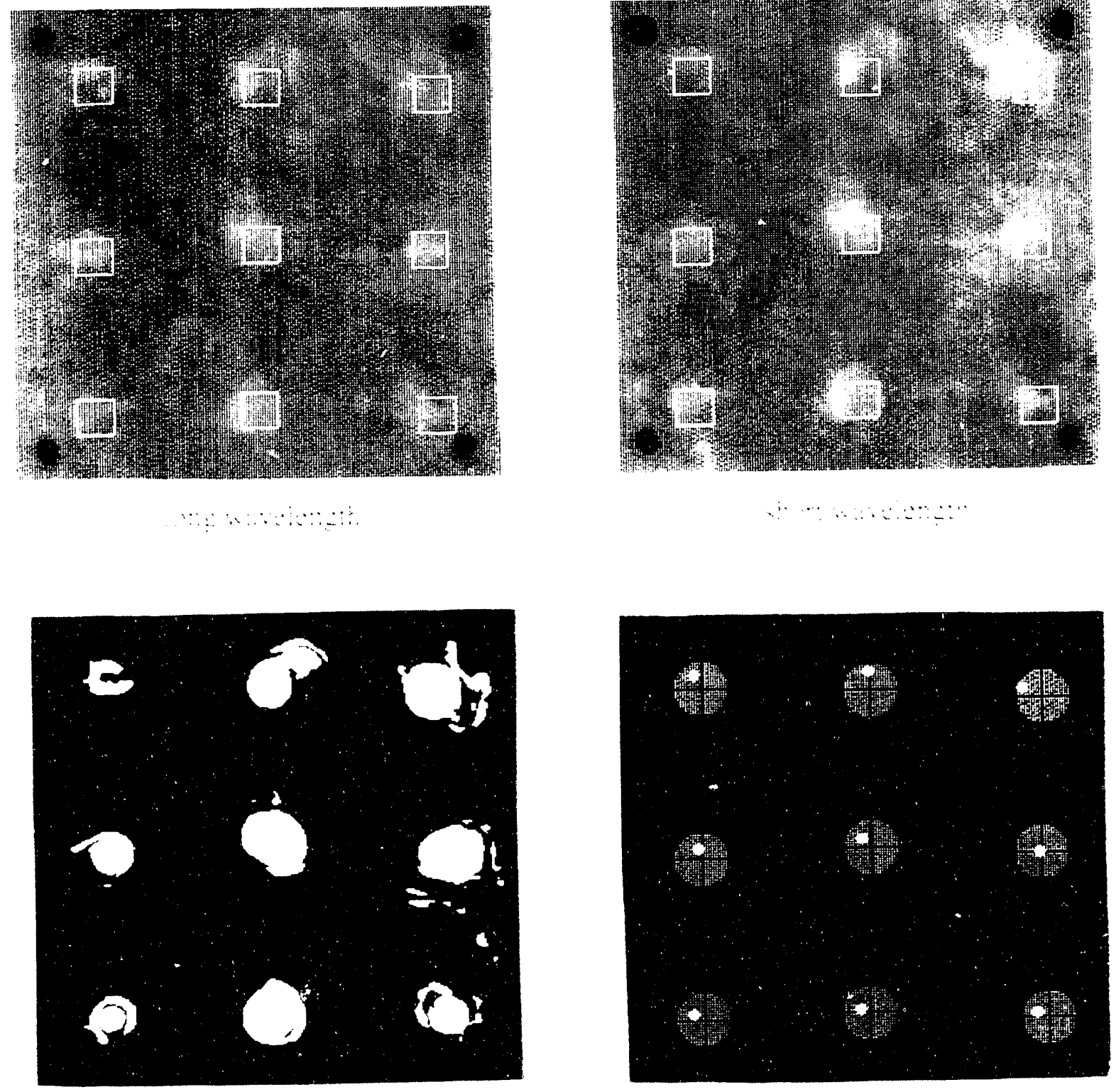


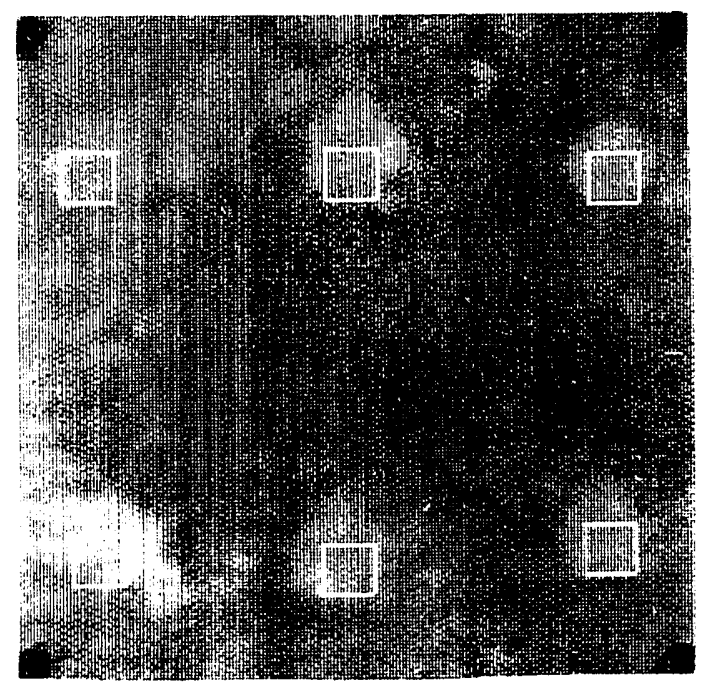

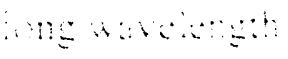

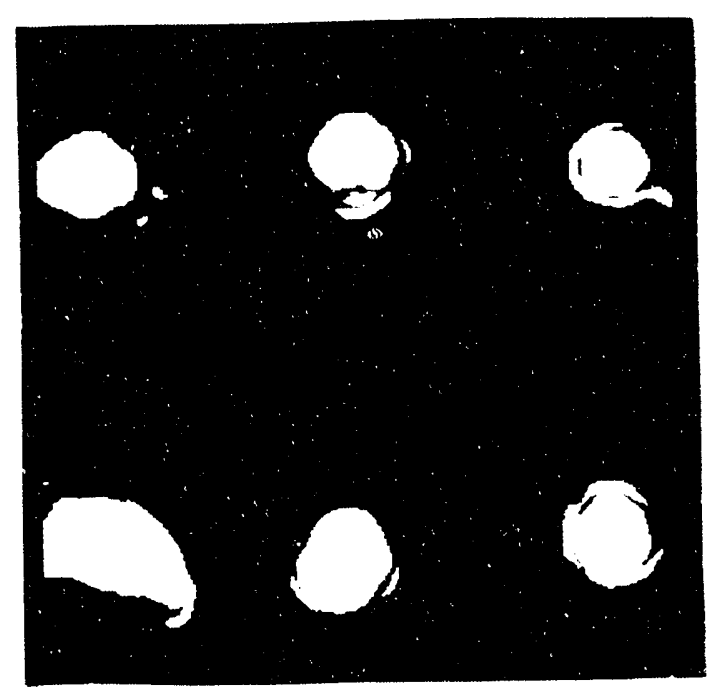

$\ldots \cdots \cdots$

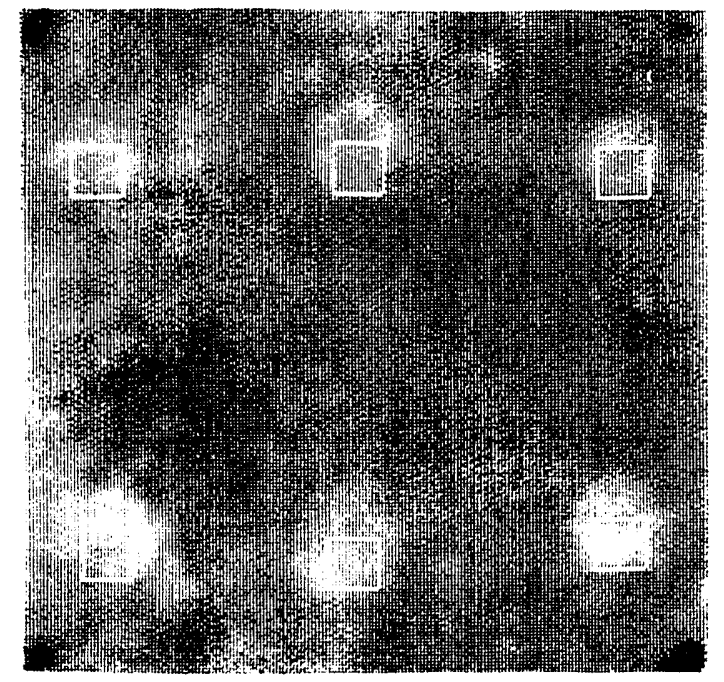

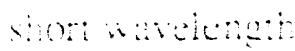

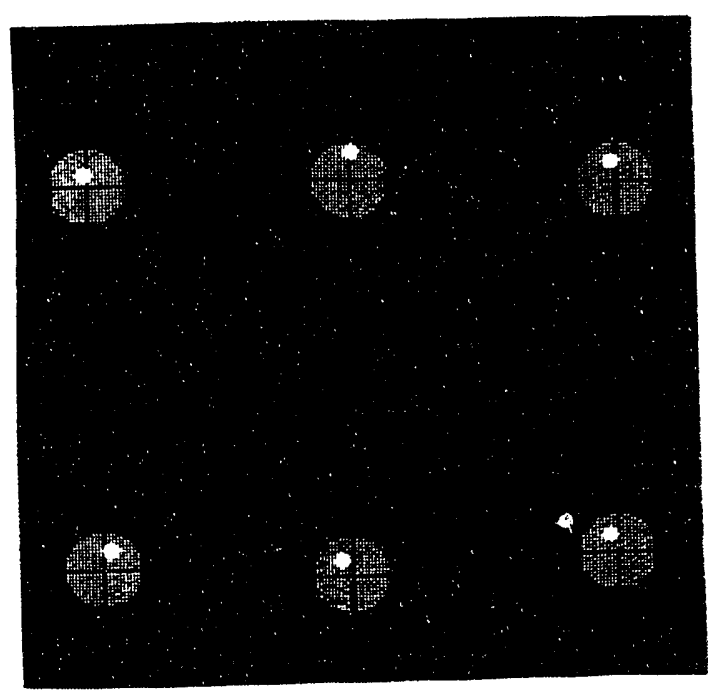

$\cdots$ 


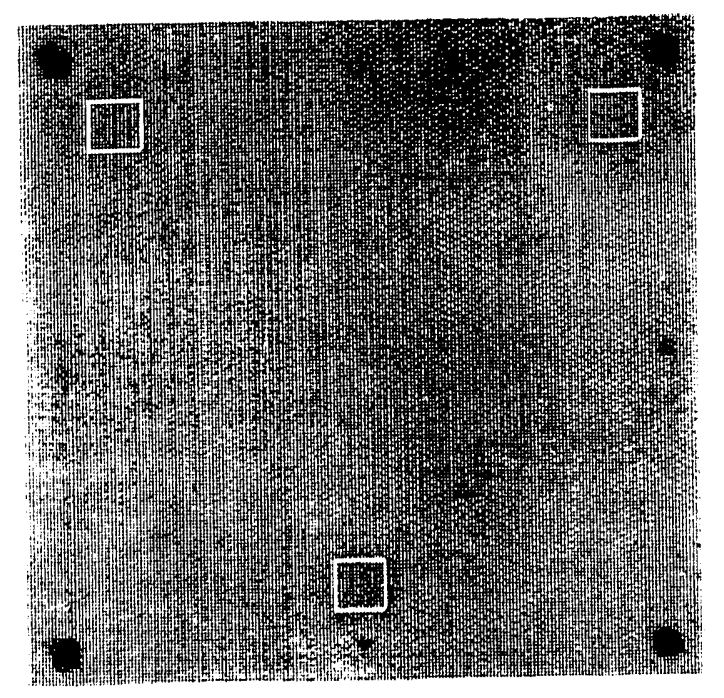

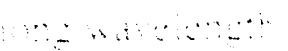


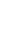




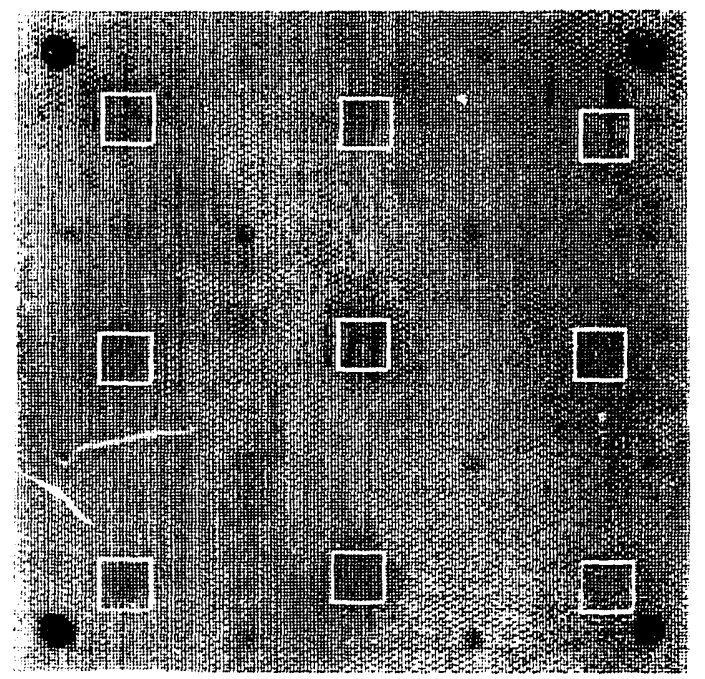

ne wawangh

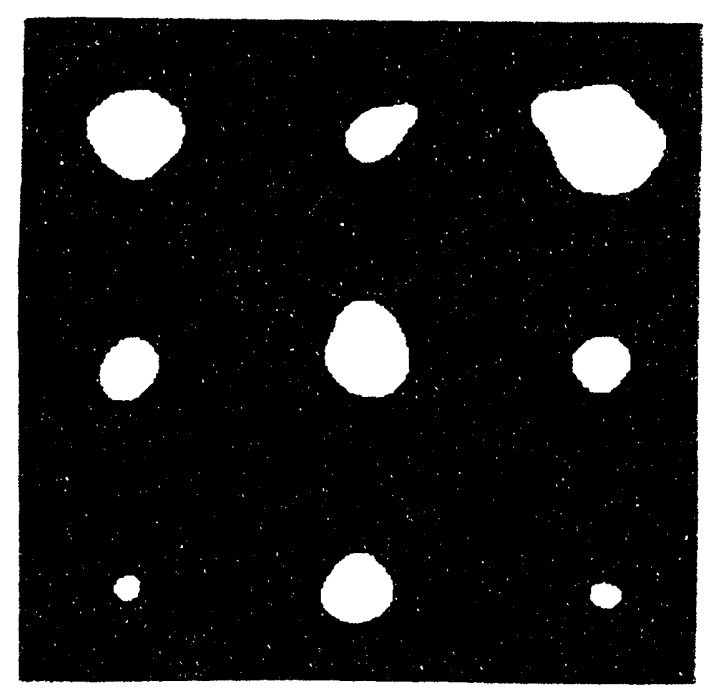

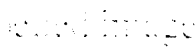

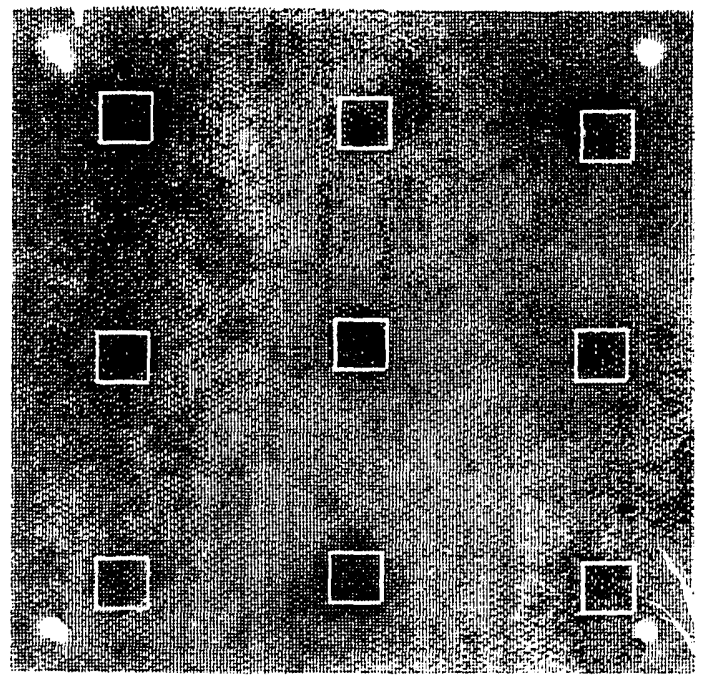

4om an: an?

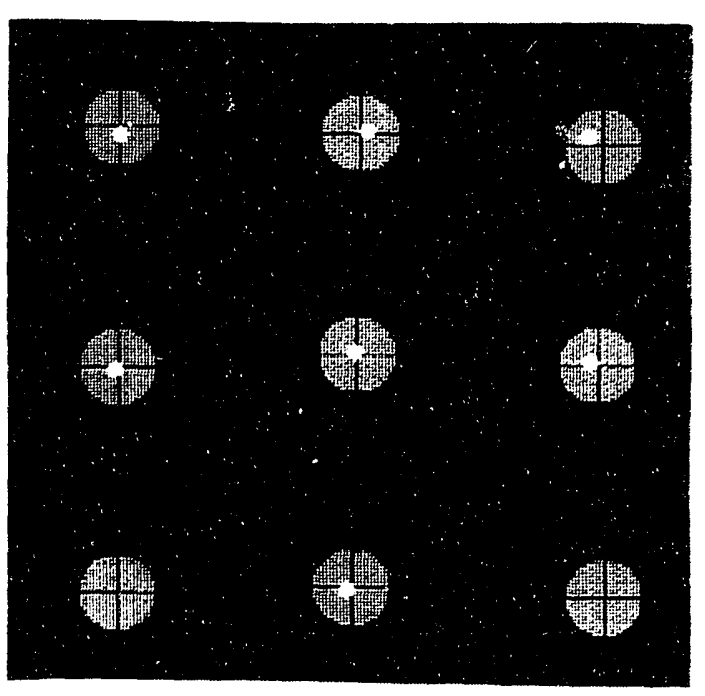

$\because \cdots$ 




$\because=4 n+a n g h$


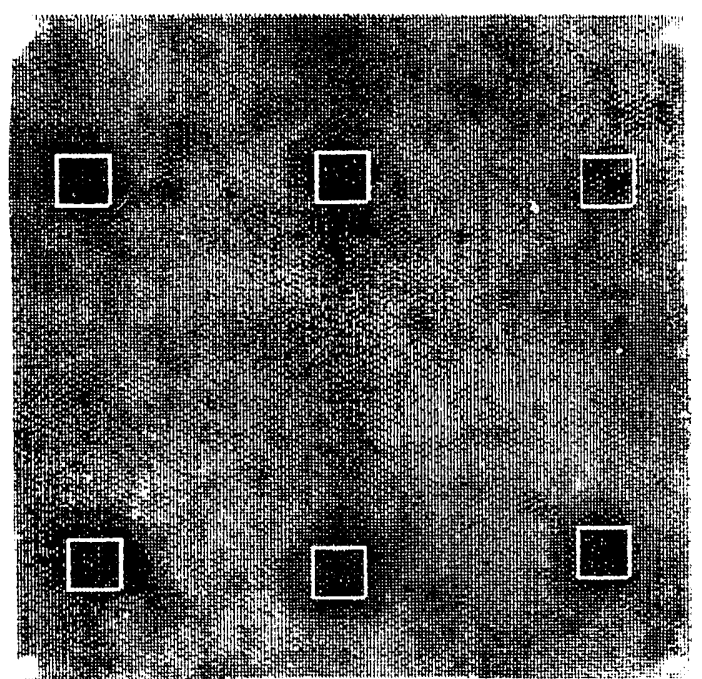

s.mon waren

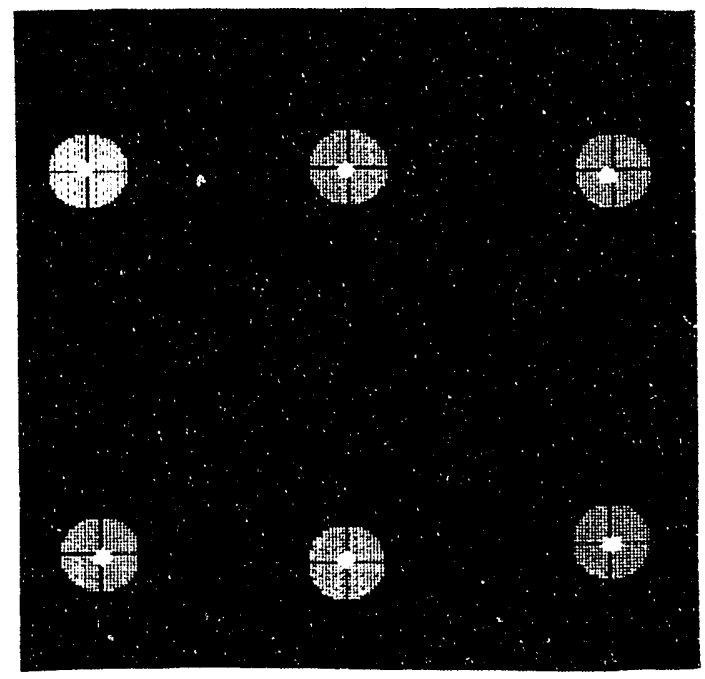

$\because \cdots$ 

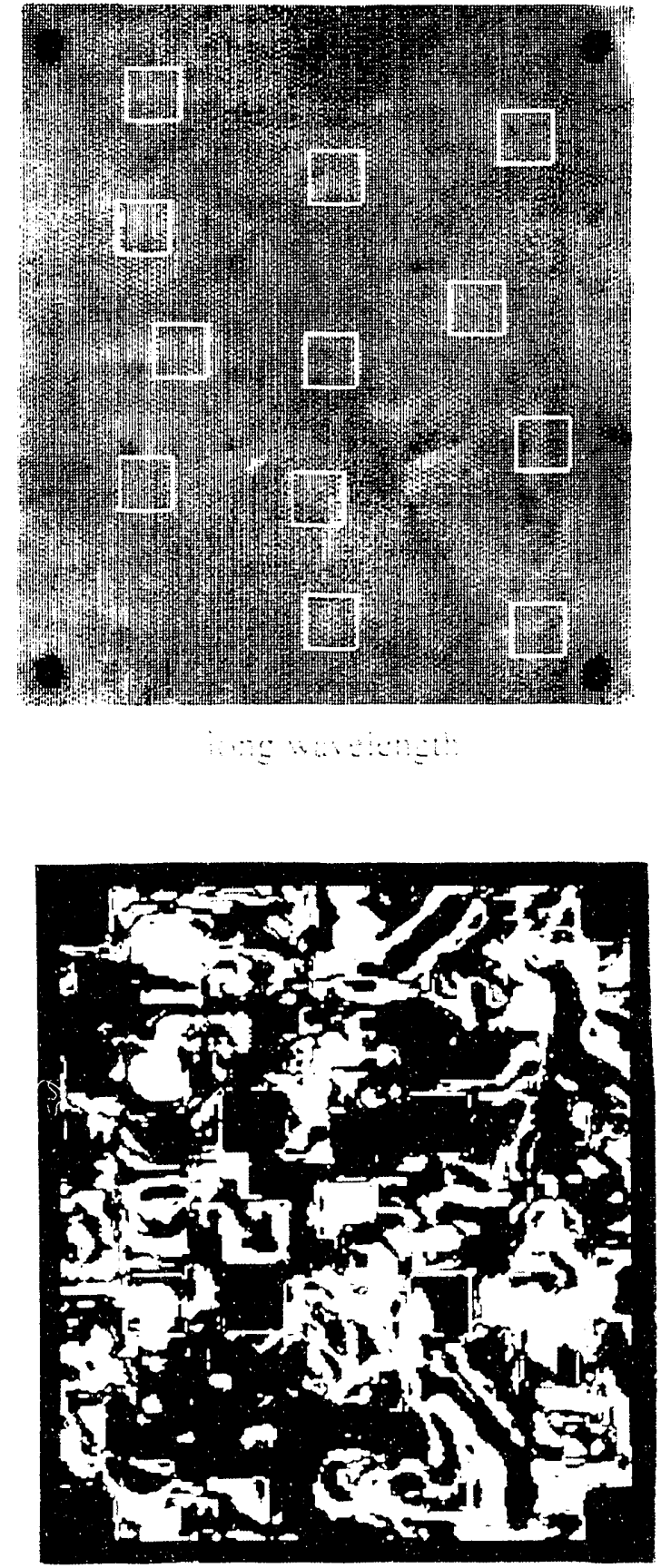
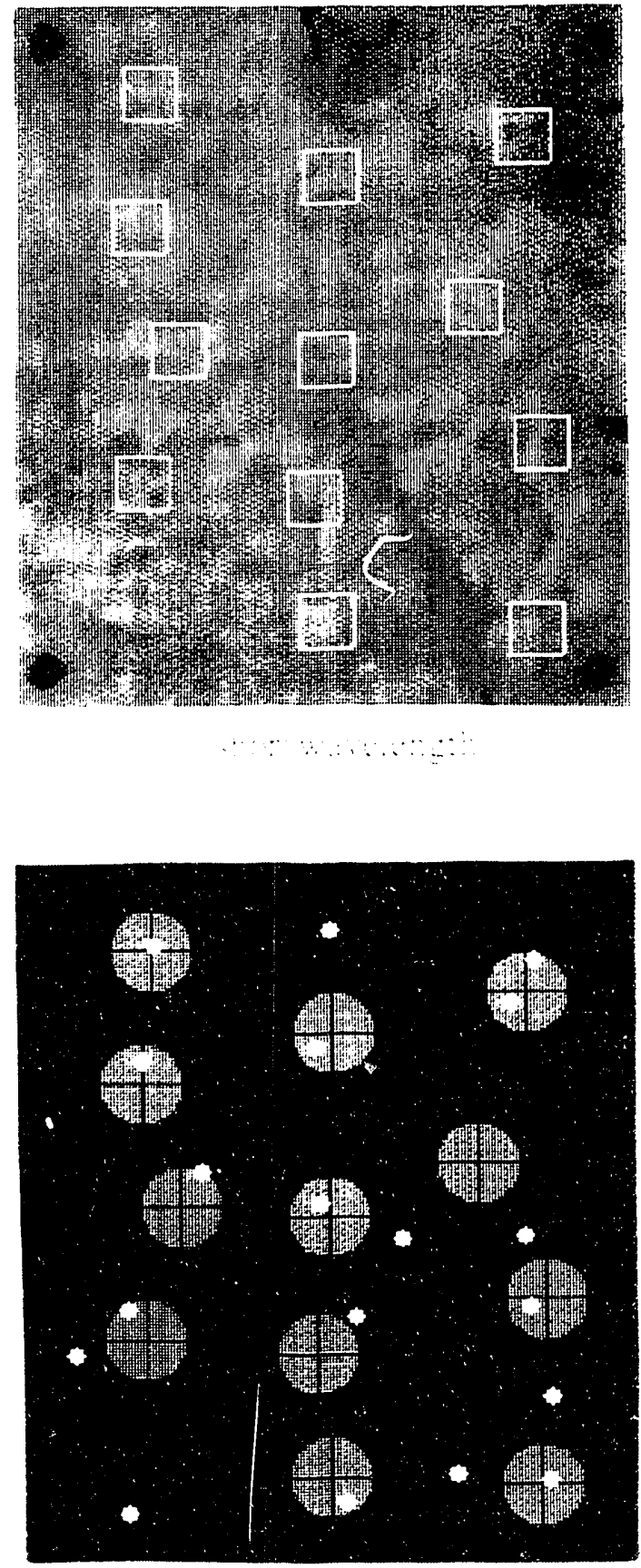

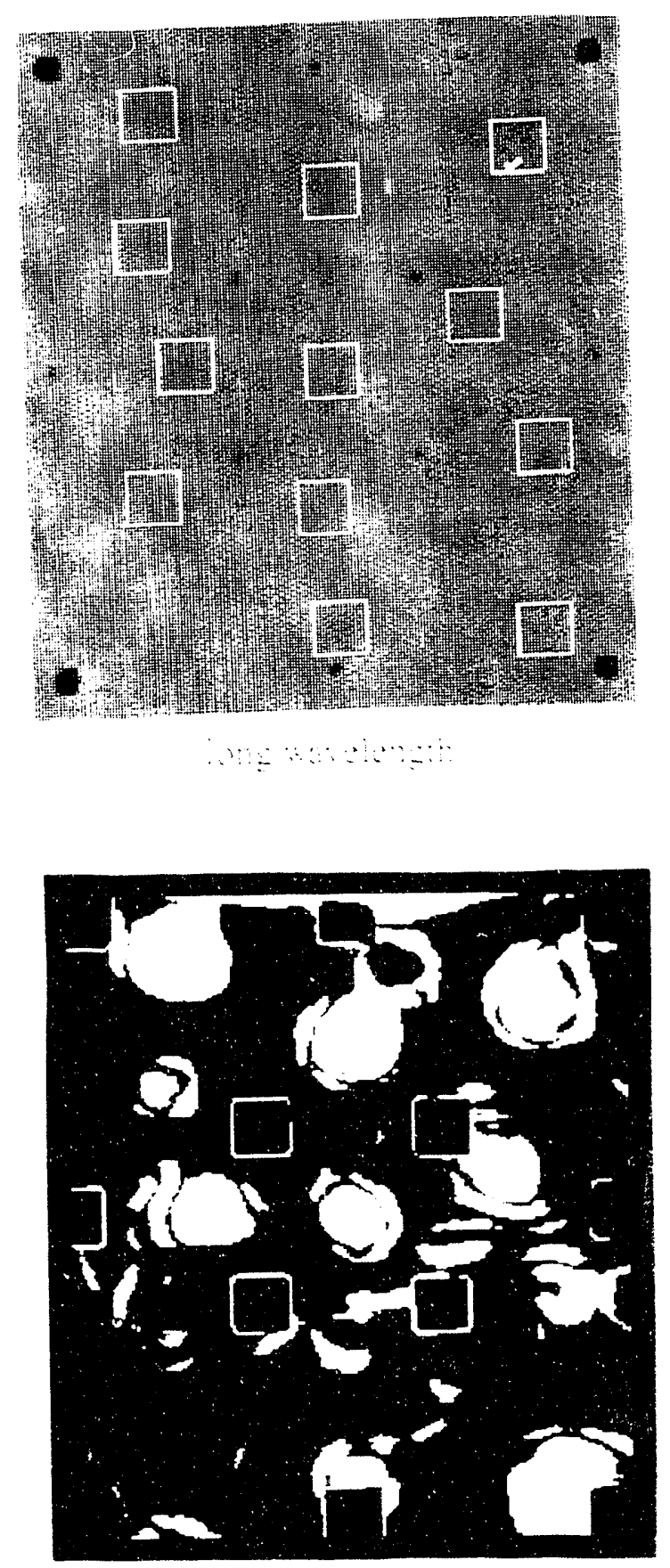



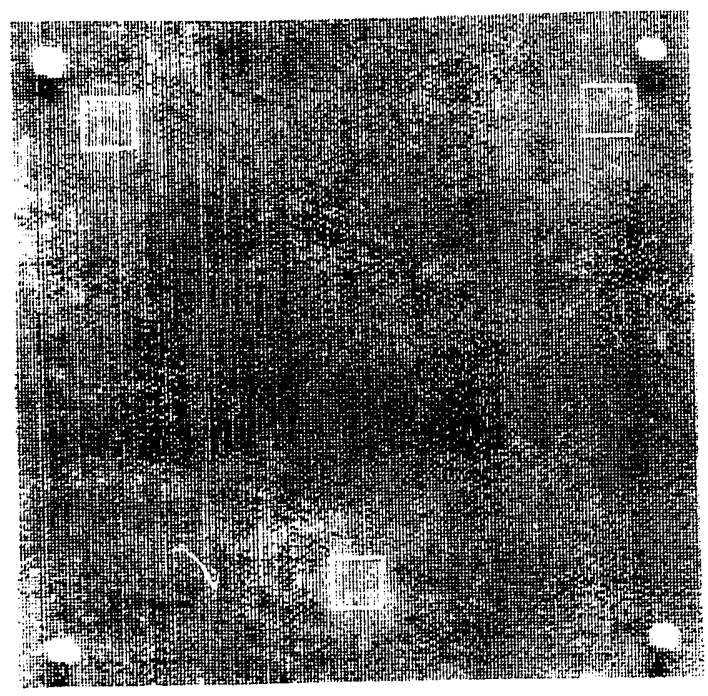

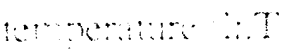

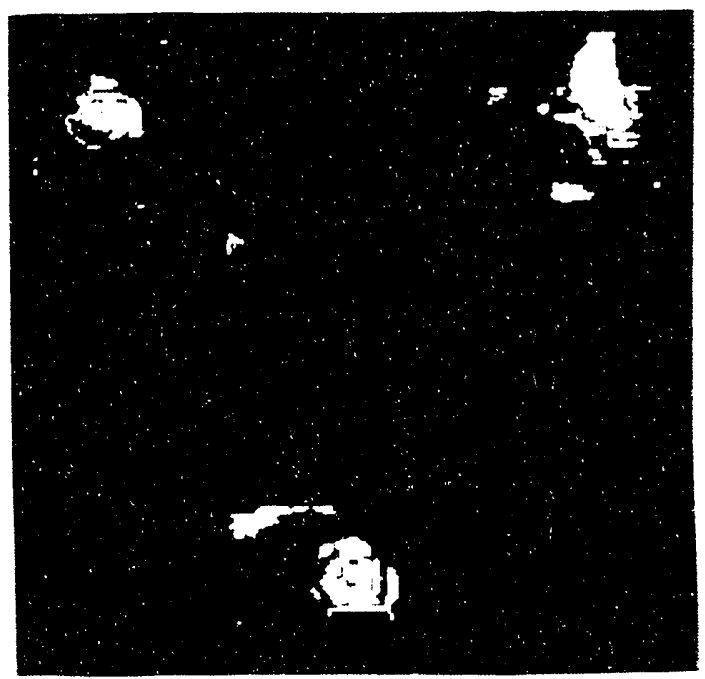

n.r.s.

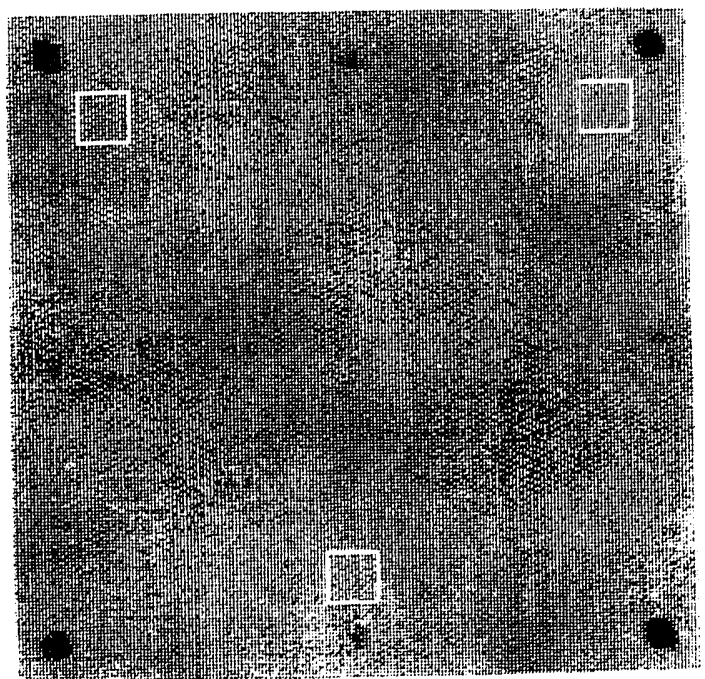

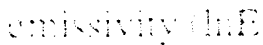

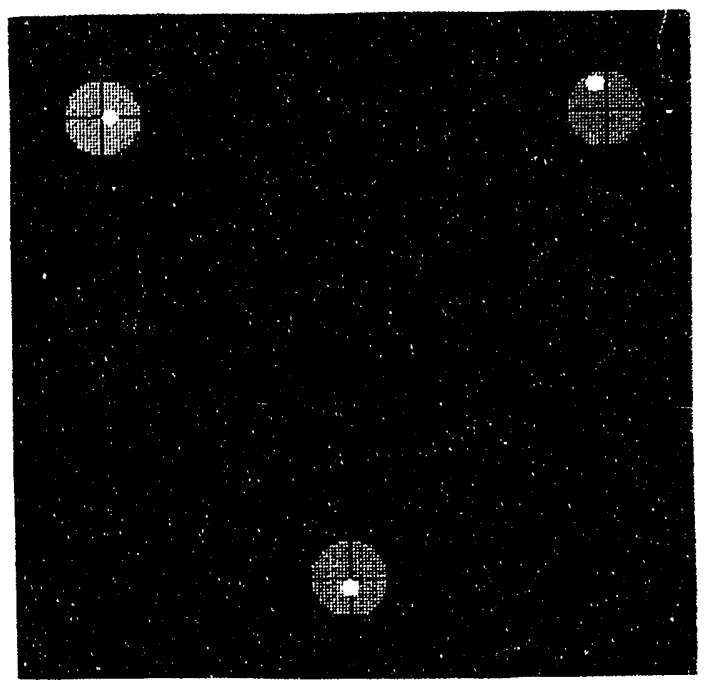

$\because \ldots$, 


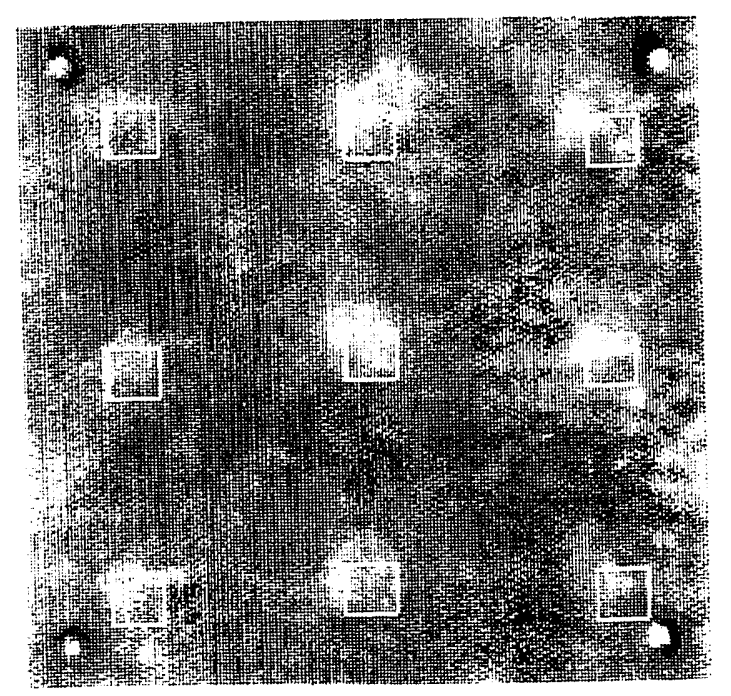

anon?

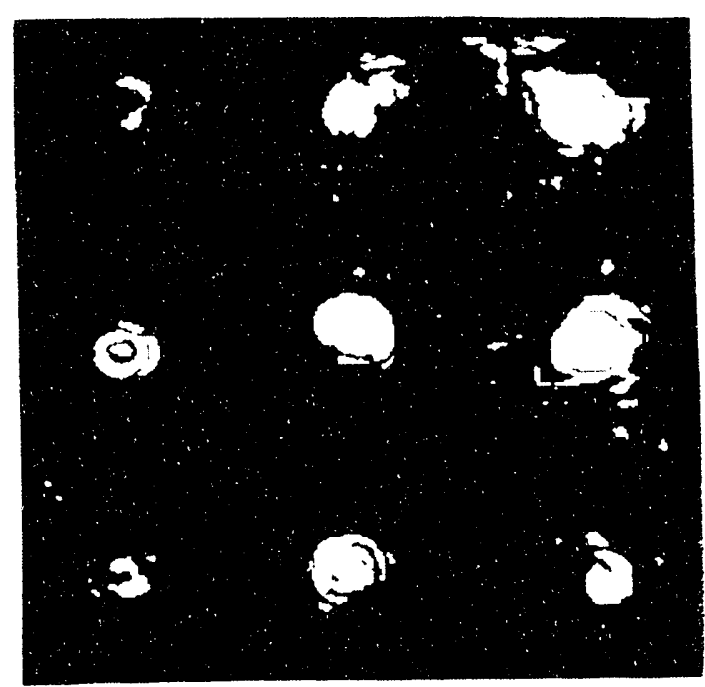

socoson

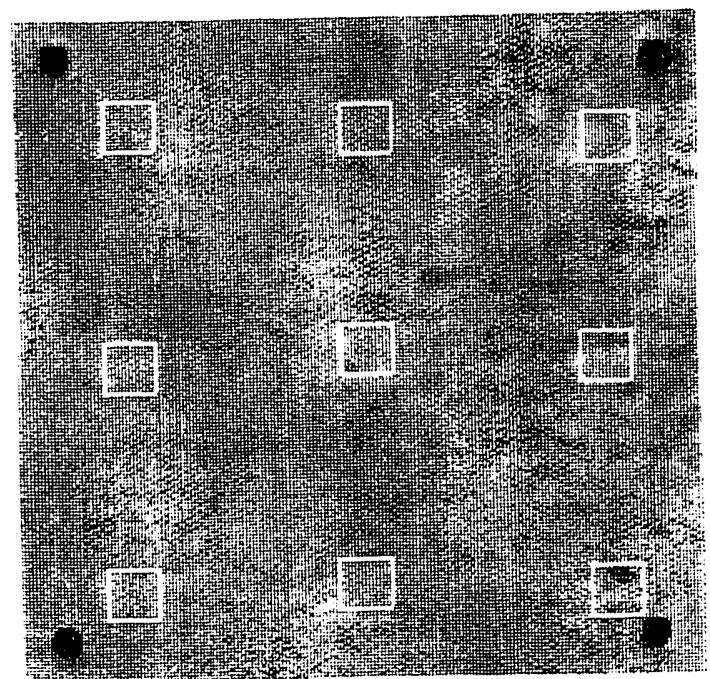

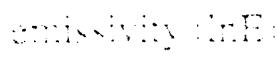
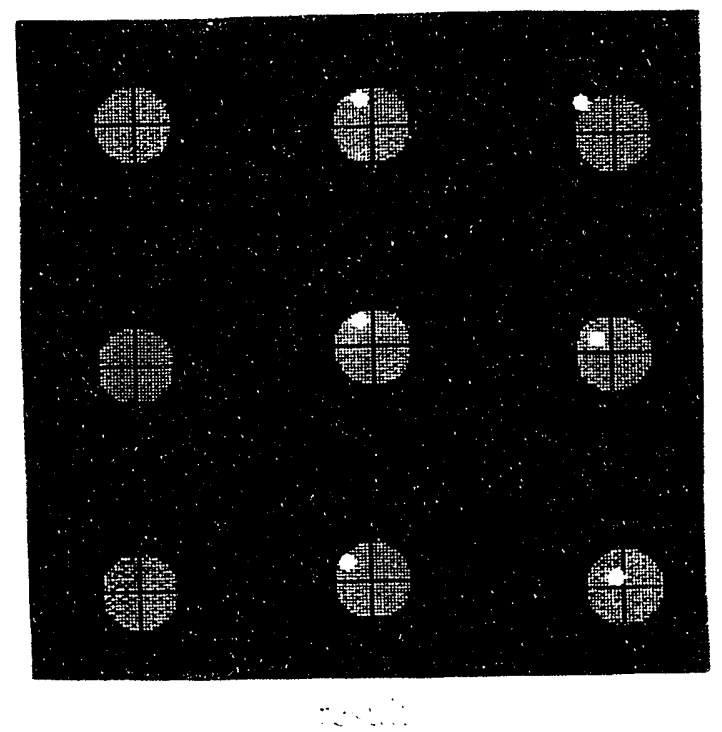


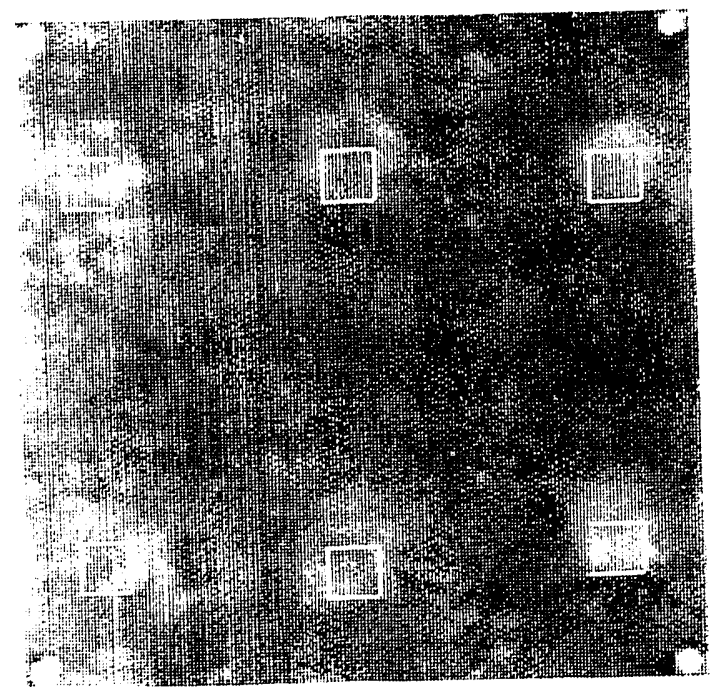

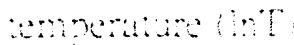

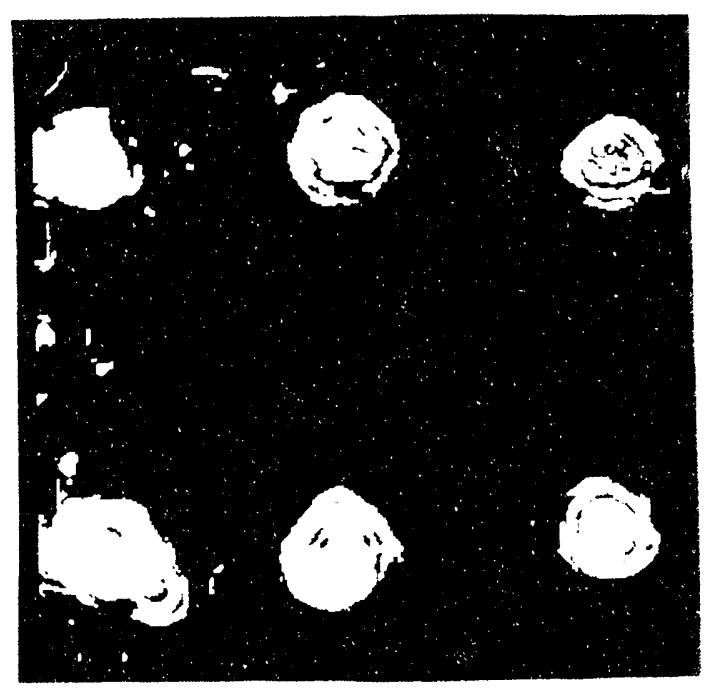

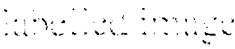

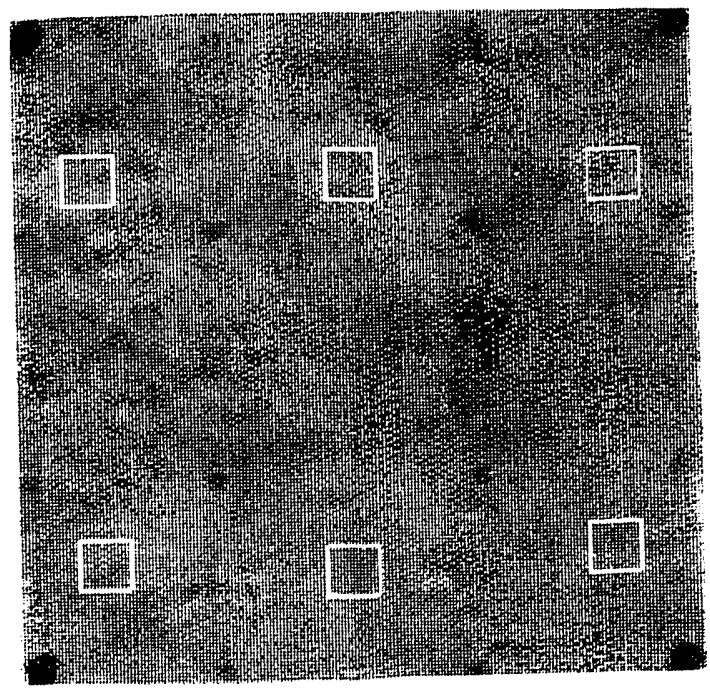

$$
\text { o.... }
$$

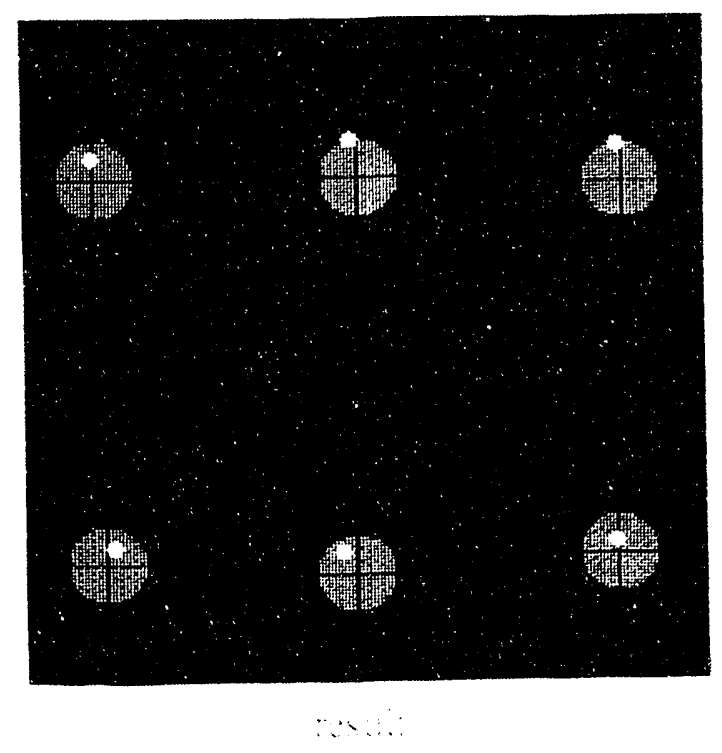




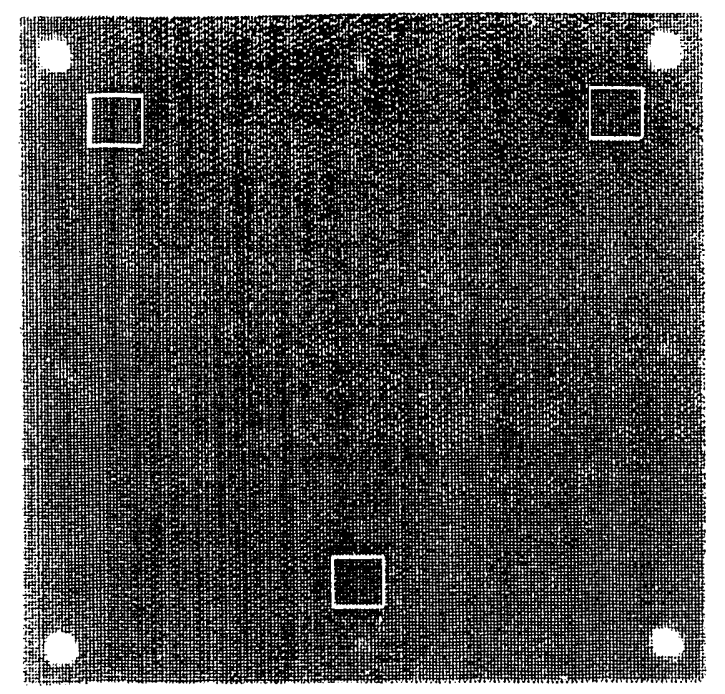

amperation :al

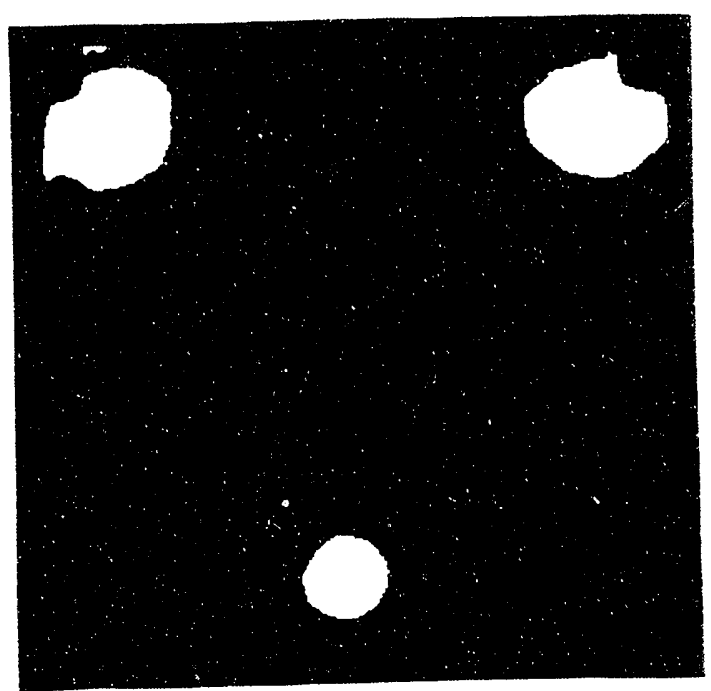

andand

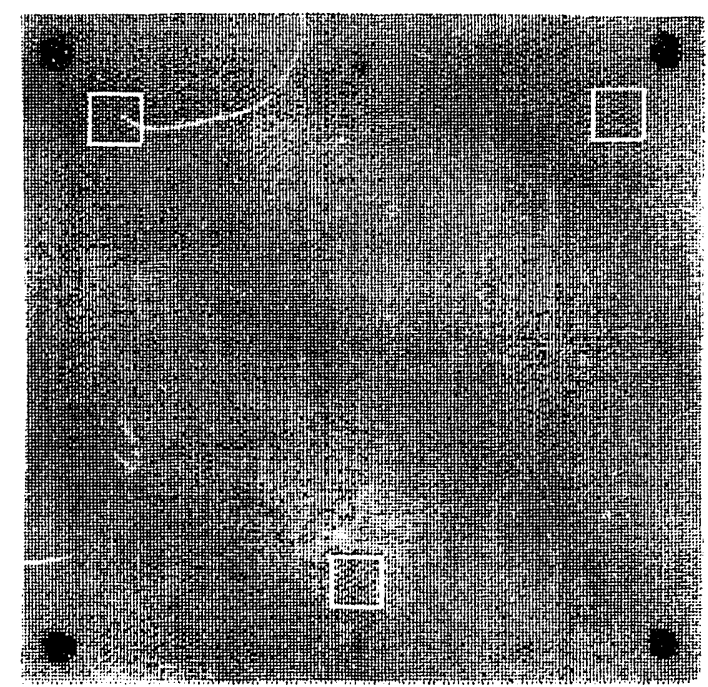

ans a

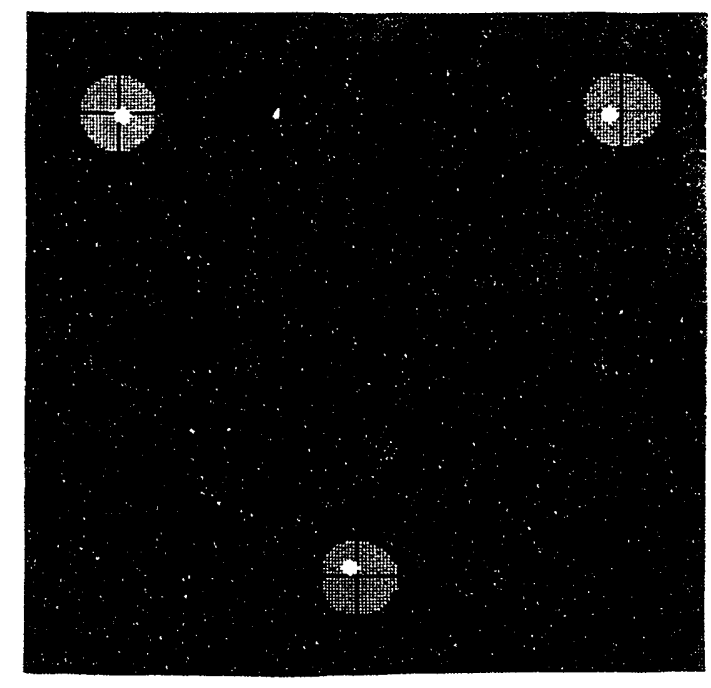

$\because \ldots$ 


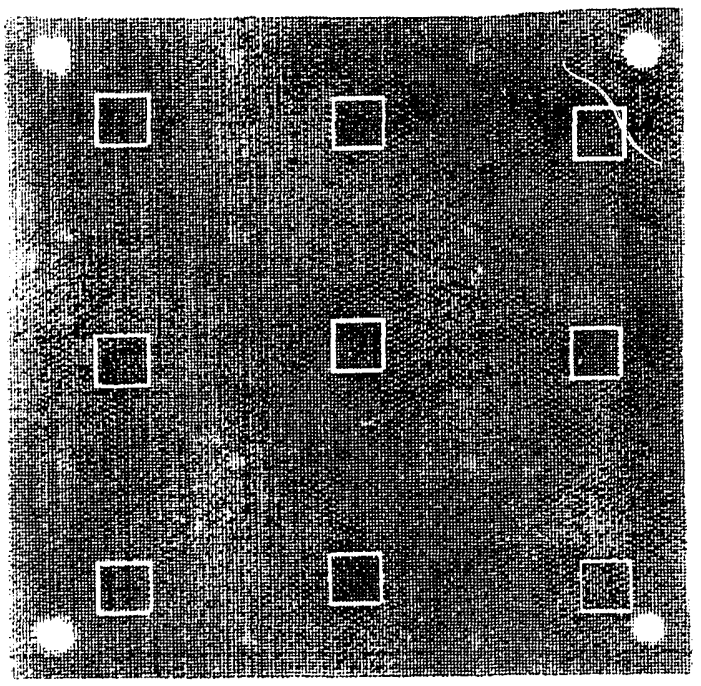

amanata ar

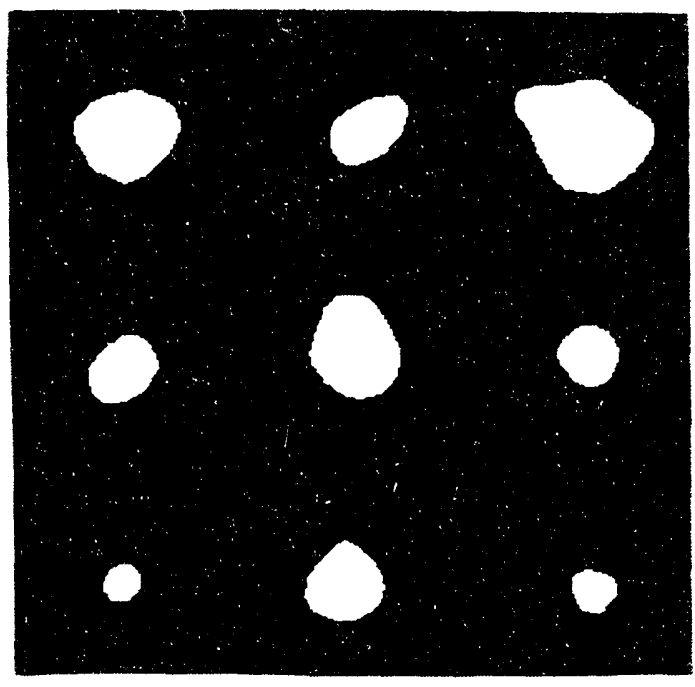

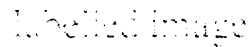

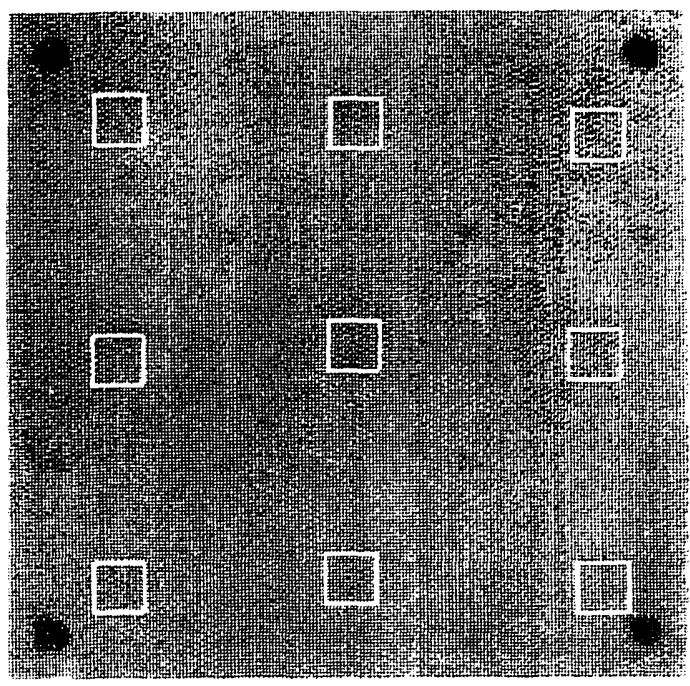

and a i :

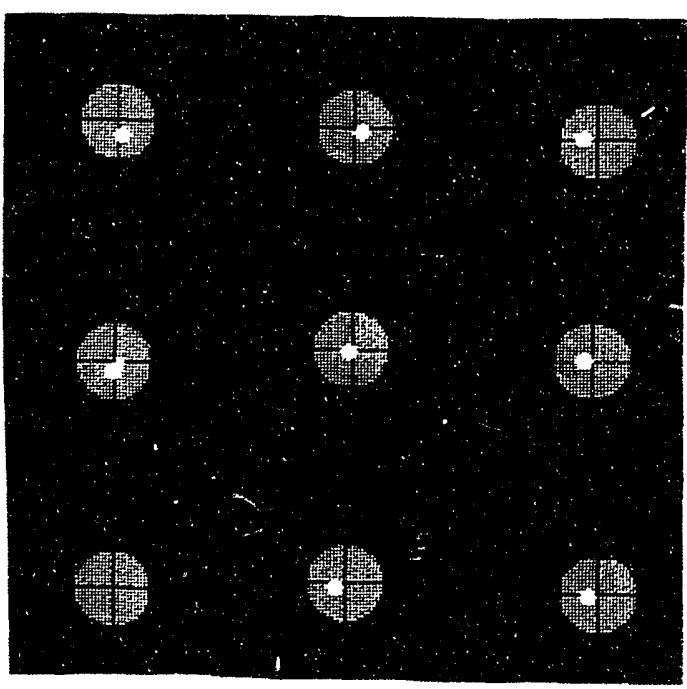

$\therefore \cdots$ 


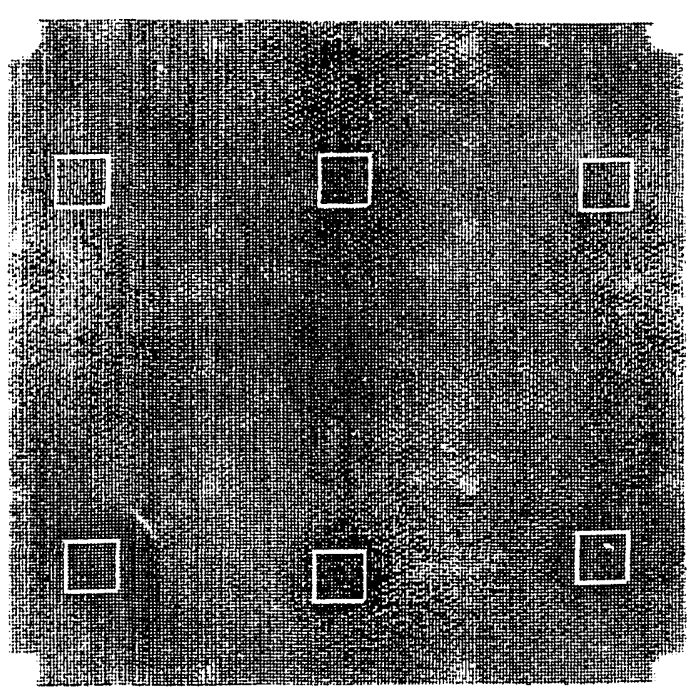



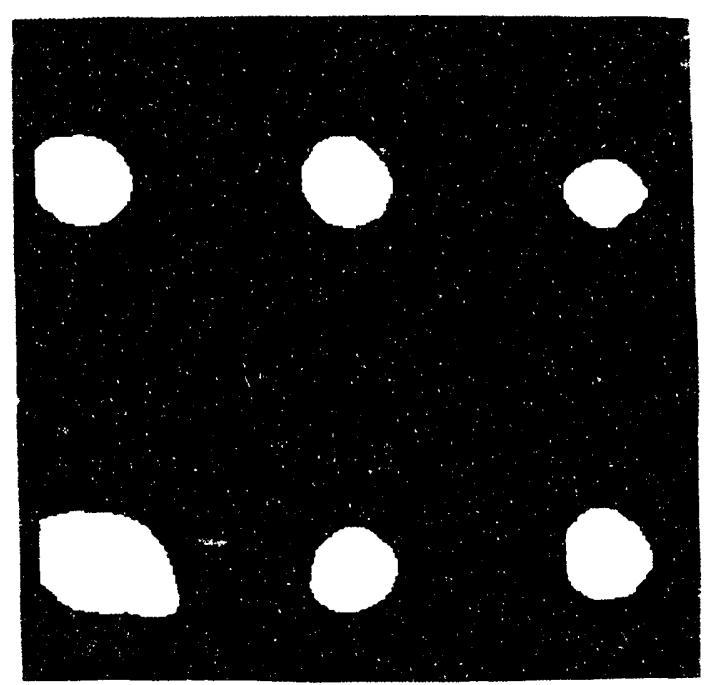

$\therefore \therefore \cdots \cdots=0$

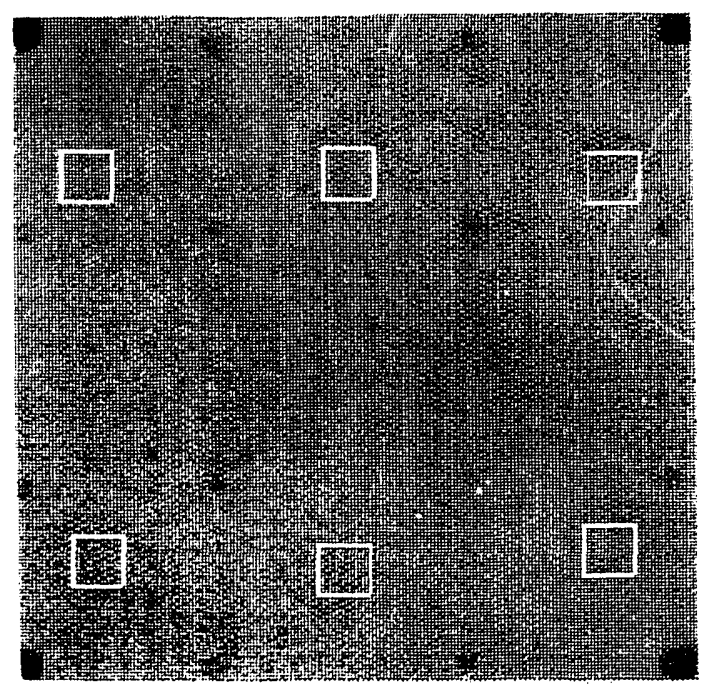

a.a

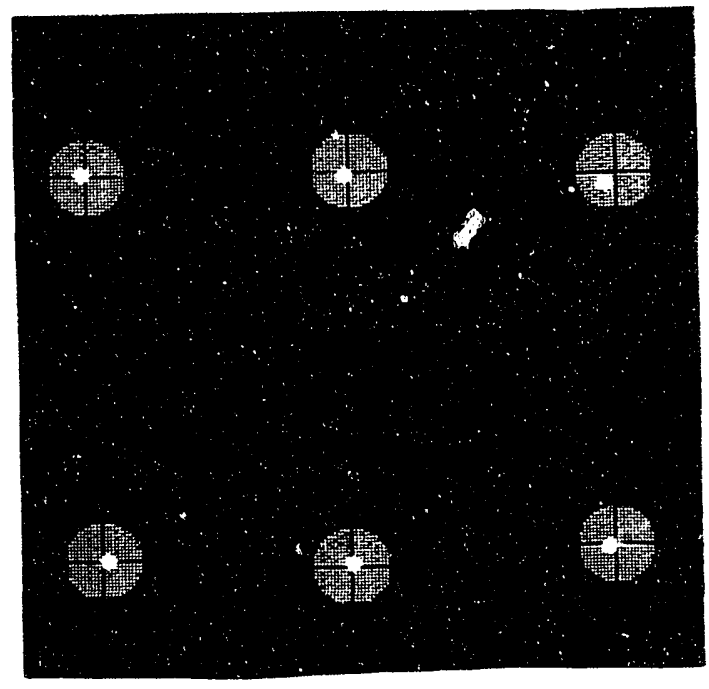

$\therefore \ldots$ 



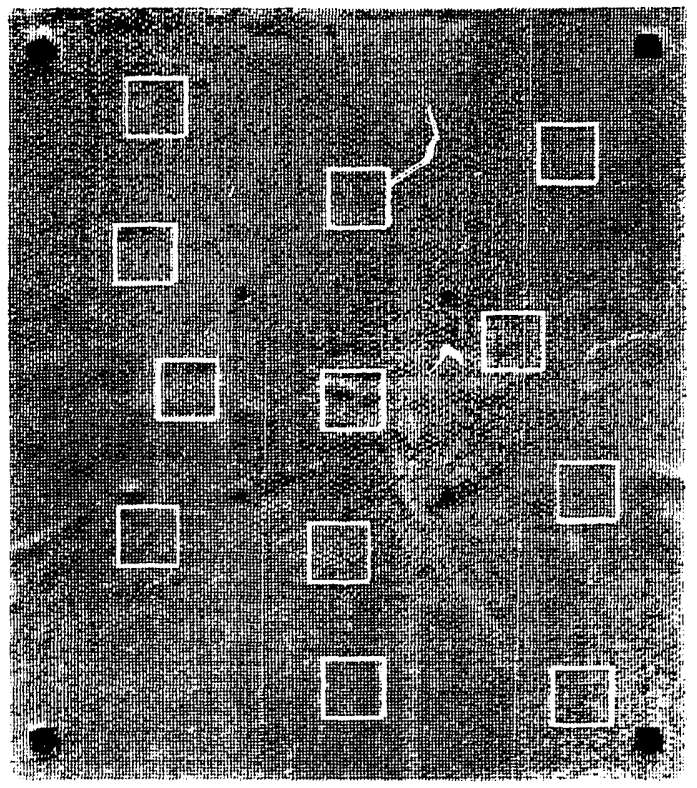

$\cdots, \cdots, \cdots$

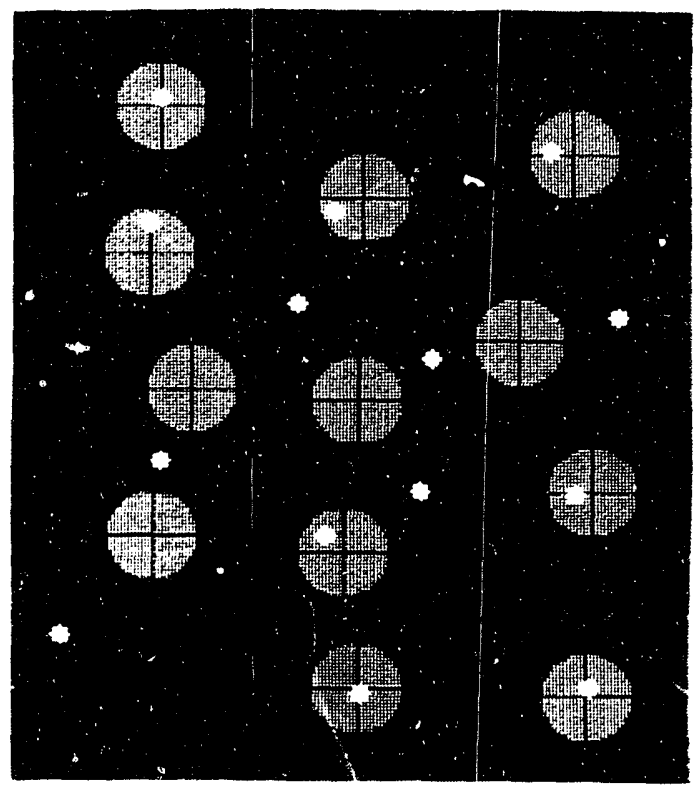




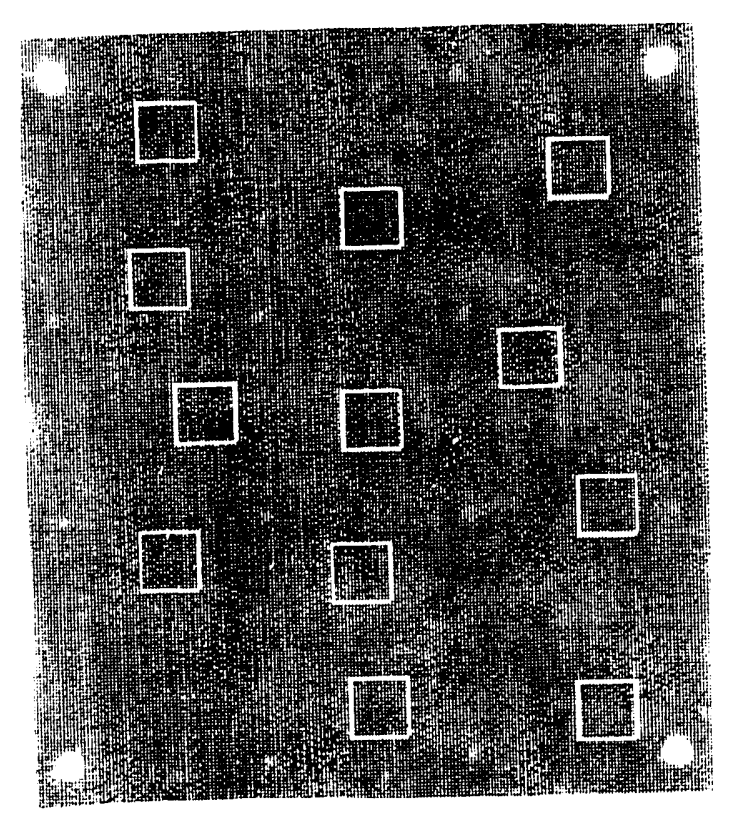

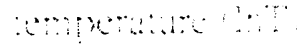
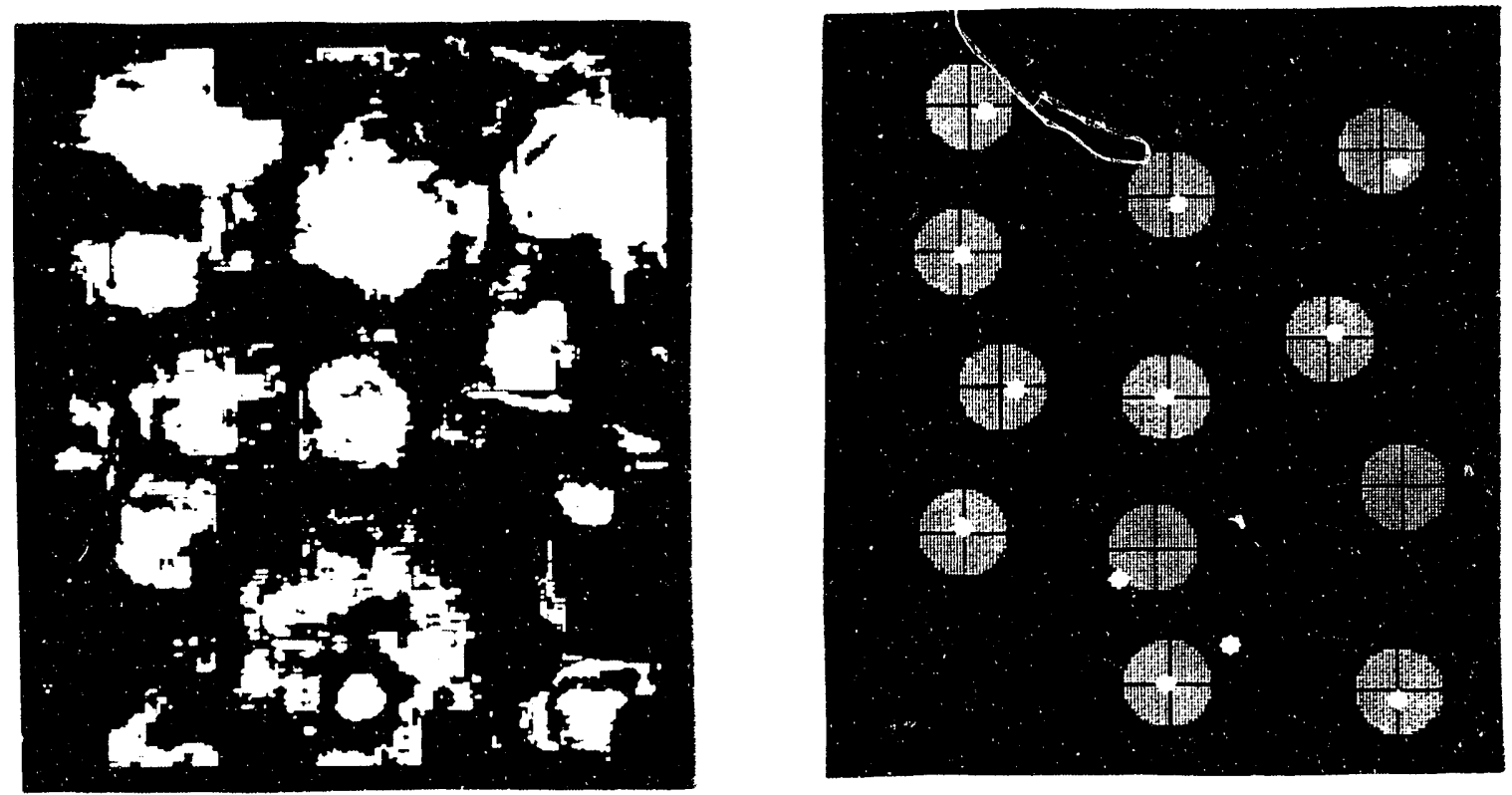


\section{REFERENCES}

[1] G.A. Clark, J.E. Hernandez, S.K. Sengupta, R.J. Sherwood, M.R. Buhl, R.J. Kane, M.J. Barth, and N.K. DelGrande, "Computer Vision and Sensor Fusion for Detecting Buried Objects", Asilomar Conference on Signals, Systems, and Computers, Pacific Grove, CA, October 26-28, 1992.

[2] G.A. Clark, J.E. Hernandez, N.K. Del Grande, R.J. Sherwood, S.Y. Lu, P.C. Schaich, and P.F. Durbin, "Computer Vision for Locating Buried Objects", Asilomar Conference on Signals, Systems, and Computers, Pacific Grove, CA, November 46, 1991.

[3] R.J. Sherwood, "Algorithm and Software Enhancement for the Temperature Evaluated Mine Position Survey Project", Thrust Area Report FY 91, Lawrence Livermore National Laboratory.

[4] N.K. Del Grande, G.A. Clark, P.F. Durbin, D.J. Fields, J.E. Hernandez, and R.J. Sherwood, "Buried Object Remote Detection Technology for Law Enforcement", SPIE Orlando 91 Symposium, Orlando, Florida, April 1-5, 1991.

[5] N.K. Del Grande, "Airborne Detection of Buried Minefields", Energy and Technology Review, December, 1991.

[6] J.E. Hernandez, M.R. Buhl, S. Sengupta, "Detecting and Locating Buried Mines from Dual-band IR Data: A Statistical Pattern Recognition Appruach", Lawrence Livermore National Laboratory.

[7] W.K. Pratt, Digital Image Processing, Second Edition. New York: Wiley, 1991, pp.559-561.

[8] R.M. Welch, K. Kuo, S.K. Sengupta, "Cloud and Surface Textural Features in Polar Regions", IEEE Trans. Geoscience and Remote Sensing, vol. 28, No. 4, pp 520-528, July 1990.

[9] T.Y. Young, K. Fu, Handbook of Pattern Recognition and Imoze Processing. Orlando: Academic Press, 1986, pp.64-74.

[10] R.O. Duda and P.E. Hart, Pattern Classification and Scene Analysis: Wiley, 1973.

[11] Anil K. Jain, Fundamentals of Digital Image Processing,. Prentice -Hall, Inc, 1989. 
[12] R.M. Haralick, L.G. Shapiro, Computer and Robot Vision, Vol. 1. AddisonWesley Publishing Company, 1992. 



$10 / 20 / 93$

